\title{
Throughput and Delay Analysis of HARQ with Code Combining over Double Rayleigh Fading Channels
}

\author{
Ali Chelli, Member, IEEE, Emna Zedini, Mohamed-Slim Alouini, Fellow, IEEE, \\ Matthias Pätzold, Senior Member, IEEE, and Ilangko Balasingham, Senior Member, IEEE
}

\begin{abstract}
This paper proposes the use of hybrid automatic repeat request (HARQ) with code combining (HARQ-CC) to offer reliable communications over double Rayleigh channels. The double Rayleigh fading channel is of particular interest to vehicle-to-vehicle communication systems as well as amplify-andforward relaying and keyhole channels. This work studies the performance of HARQ-CC over double Rayleigh channels from an information theoretic perspective. Analytical approximations are derived for the $\epsilon$-outage capacity, the average number of transmissions, and the throughput of HARQ-CC. Moreover, we evaluate the delay experienced by Poisson arriving packets for HARQ-CC. We provide analytical expressions for the average waiting time, the packets sojourn time, the average consumed power, and the energy efficiency. In our investigation, we take into account the impact of imperfect feedback on different performance metrics. Additionally, we explore the tradeoff between energy efficiency and the throughput. The proposed scheme is shown to maintain the outage probability below a specified threshold $\epsilon$ which ensures the link reliability. Meanwhile, HARQCC adapts implicitly the transmission rate to the channel conditions such that the throughput is maximized. Our results demonstrate that HARQ-CC allows improving the achievable communication rate compared to fixed time diversity schemes. To maximize the throughput of HARQ-CC, the rate per HARQ round should be less than the rate required to meet the outage constraint. Our investigation of the performance of HARQ-CC over Rayleigh and double Rayleigh channels shows that double Rayleigh channels have a higher severity of fading and result in a larger degradation of the throughput. Our analysis reveals that HARQ with incremental redundancy (HARQ-IR) achieves a larger throughput compared to HARQ-CC, while HARQ-CC is simpler to implement, has a lower decoding complexity, and requires less memory resources.
\end{abstract}

Index Terms-Vehicle-to-vehicle communications, double Rayleigh channel, hybrid automatic repeat request (HARQ), code combining, throughput, delay analysis, energy efficiency, information outage capacity.

Copyright (c) 2015 IEEE. Personal use of this material is permitted. However, permission to use this material for any other purposes must be obtained from the IEEE by sending a request to pubs-permissions@ieee.org.

A. Chelli and M. Pätzold are with the Faculty of Engineering and Science, University of Agder, 4898 Grimstad, Norway (e-mails:\{ali.chelli, matthias.paetzold\}@uia.no).

E. Zedini is with the College of Science and Engineering Hamad Bin Khalifa University (HBKU), Doha, Qatar (e-mail:ezedini@hbku.edu.qa).

M.-S. Alouini is with the Computer, Electrical, and Mathematical Science and Engineering (CEMSE) Division, King Abdullah University of Science and Technology (KAUST) Thuwal, Makkah Province, Saudi Arabia (email:slim.alouini@kaust.edu.sa).

I. Balasingham is with the Department of Electronics and Telecommunications, Norwegian University of Science and Technology (NTNU), 7491 Trondheim, Norway (e-mail:ilangko.balasingham@ntnu.no).

This work is an extended version of a paper published in the Proceedings of the IEEE 22nd Symposium on Personal, Indoor and Mobile Radio Communications (PIMRC 2011), Toronto, Canada, September 2011.

\section{INTRODUCTION}

\section{A. Background}

Double Rayleigh fading channels represent an important category of communication channels. The fading distribution is modelled as double Rayleigh if the transmitted signal undergoes a cascaded Rayleigh fading. The study of double Rayleigh fading channels has gained a lot of interest due to its applicability to various communication scenarios. These communication scenarios include vehicle-to-vehicle (V2V) channels, amplify-and-forward relaying, and keyhole channels.

$\mathrm{V} 2 \mathrm{~V}$ measurement results in [1] have shown that in urban environments, the double-bounce scattering components caused by fixed scatterers are dominant. The double-bounce scattering mechanism has been assumed in several V2V channel models [2]-[4]. Under non-line-of-sight assumptions, the double-bounce scattering mechanism results in a cascaded Rayleigh channel. For such a channel, the probability density function (PDF) of the envelope follows the double Rayleigh distribution. Thus, the double Rayleigh distribution is a good fading model especially for urban $\mathrm{V} 2 \mathrm{~V}$ channels.

Moreover, for amplify-and-forward relaying systems if the source-relay link and the relay-destination link are modelled each by a Rayleigh distribution, the end-to-end link from the source to the destination follows a double Rayleigh distribution [5]. In addition, the double Rayleigh distribution is a wellaccepted model for keyhole channels. For such channels, the transmitted signal reaches the receiver only through an electromagnetically small aperture called a keyhole. If both the transmitter and the receiver are moving and the number of scatterers around each of them is large, a keyhole channel is created and the fading envelope follows a double Rayleigh distribution [6], [7]. It has been shown in [8] that the severity of fading is larger for double Rayleigh channels compared to the classical Rayleigh channel. Therefore, it is highly important to propose communication techniques which can improve the link reliability over double Rayleigh channels.

In order to provide reliable data transmission, error control mechanisms are absolutely essential in communication systems. Hybrid automatic repeat request (HARQ) represents one of the main error control techniques. HARQ combines both forward error correction and automatic repeat request (ARQ) [9]. HARQ is widely used in contemporary communication systems, such as High Speed Packet Access (HSPA) and Long Term Evolution (LTE) [10] and can also be used to offer reliable communication over double Rayleigh channels. There are mainly two main categories of HARQ schemes, namely, 
HARQ type-I and HARQ type-II. For HARQ type-I, a packet is transmitted over the wireless link and if the reception of the packet fails, the received copy of the packet is discarded and negative acknowledgement (NACK) is sent to the transmitter. This procedure is repeated until successful reception occurs or a maximum number $M$ of rounds is reached. As opposed to HARQ type-I, for HARQ type-II the copies of the same packet received over different HARQ rounds are not discarded in case of reception failure. These copies are stored and combined with the copies of the same packet received in subsequent HARQ rounds. These combined copies are then used for decoding purpose, which makes HARQ type-II more robust against fading and increases its throughput. In HARQ typeII, there are mainly two schemes, namely, HARQ with code combining (HARQ-CC), which has been proposed by Chase in [11] and HARQ with incremental redundancy (HARQIR). In the HARQ-CC scheme, the same packet is resent until successful reception or a maximum number of rounds $M$ is reached. At the receiver side, all received bits are combined together before the start of the decoding process. In this setting, the time diversity results in a more reliable communication. The receiver sends an ACK/NACK message to indicate successful/unsuccessful reception.

\section{B. Related Work}

Several research papers have focused on information theoretic aspects of HARQ. In [12], the authors have derived a relationship between HARQ throughput and HARQ mutual information. A three-dimensional tradeoff between reliability, throughput, and delay for MIMO ARQ systems is introduced in [13]. The diversity-multiplexing-delay tradeoff was studied in [13] from an information theoretic perspective over Gaussian block fading channels. The outage probability and the long terms average throughput of HARQ-IR over temporally correlated Rayleigh fading channels have been investigated in [14]. The throughput of different ARQ schemes over Gaussian block fading channels has been analysed in [15]. An adaptive power allocation algorithm has been proposed in [16] for systems using HARQ-IR. Under quality-of-service constraints and limited feedback, the proposed algorithm allows achieving sub-optimal performance with reasonable complexity. In [17], the performance of both HARQ-IR and HARQ-CC over relay networks has been studied. In [18], it has been shown that, for a fixed outage probability, HARQ allows to communicate at a higher rate compared to a fixed time diversity scheme in the case of Rayleigh block fading channels. The amount of information sent through the channel is adapted implicitly to the link quality even in absence of channel state information (CSI) at the transmitter. This feature makes HARQ very attractive, especially for fast fading channels, such as V2V channels.

\section{Contributions}

The objective of this work is to investigate the performance of HARQ-CC with a fixed outage probability. The main contributions of this work are listed as follows.
- We provide a generic analysis for HARQ-CC without assuming a prior fading channel distribution.

- We analyze the performance of HARQ-CC over both Rayleigh and double Rayleigh channels and provide analytical expressions for the throughput, the $\epsilon$-outage capacity and the average number of transmissions of HARQ-CC.

- We formulate the throughput optimization problem and determine the optimal rate per round such that the throughput of HARQ-CC is maximized.

- We show that in order to maximize the throughput, it is needed to use a rate per round that is smaller than the rate required to meet the outage constraint $\epsilon$.

- We compare the performance of HARQ-CC to a fixed time diversity scheme and illustrate the significant throughput gain achieved by the use of HARQ-CC.

- We explore the performance of HARQ-CC over Rayleigh and double Rayleigh channels. We show that the severity of fading in the case of double Rayleigh channels leads to a significant degradation in the throughput compared to classical Rayleigh channels.

- We compare the performance of HARQ-CC and HARQIR over double Rayleigh channels. We show that HARQIR has a larger throughput, but HARQ-CC is simpler to implement, has a lower decoding complexity, and requires less memory resources.

- We analyze the performance of HARQ-CC with imperfect feedback taking into account acknowledgment feedback errors. In this realistic setup, we characterize the average number of transmissions and the throughput.

- We study the energy efficiency of HARQ-CC and characterize the tradeoff between the energy efficiency and the throughput over double Rayleigh channels.

- We derive analytical expressions for the PDF of the instantaneous signal-to-noise ratio (SNR), the outage probability, and the ergodic capacity of double Rayleigh channels.

- We analyze the delay performance of HARQ-CC and derive analytical expressions for the average waiting time for the packet (time elapsed between the first packet transmission and the packet arrival) and the average sojourn time in the buffer for perfect and imperfect feedback.

The remainder of the paper is organized as follows. Section II investigates some characteristic quantities of double Rayleigh channels. Section III conducts generic analysis of HARQ-CC before deriving analytical expressions for the $\epsilon$-outage capacity, the average number of transmissions, and the throughput over Rayleigh and double Rayleigh channels. In Section IV, we formulate the throughput optimization problem of HARQ$\mathrm{CC}$ and determine the optimal rate per HARQ round that maximizes the throughput. In Section V, the performance of the system with an imperfect feedback channel is explored, while the delay model is studied in Section VI, and the energy efficiency is investigated in Section VII. The derived analytical expressions are numerically evaluated, illustrated, and interpreted in Section VIII. Finally, Section IX provides 
some concluding remarks.

\section{Characteristic Quantities of Double Rayleigh CHANNELS}

In this section, we derive some characteristic quantities of double Rayleigh channels, such as the outage probability, the ergodic capacity. We first derive an expression for the outage probability of double Rayleigh channels. To this end, we denote by $\chi(t)$ the squared envelope of a double Rayleigh process. This term is written as $\chi(t)=\chi_{11}(t) \chi_{12}(t)$, where $\chi_{11}(t)$ and $\chi_{12}(t)$ are the squared envelopes of two independent Rayleigh processes ${ }^{1}$. For a double Rayleigh channel, the instantaneous received SNR is expressed as

$$
\eta=\chi \gamma_{s}=\chi_{11} \chi_{12} \gamma_{s}
$$

In (1), $\gamma_{s}$ is defined as $\gamma_{s}=E_{s} / N_{0}$, where $E_{s}$ denotes the symbol energy and $N_{0} / 2$ is the noise power spectral density.

The outage probability $P_{\text {out }}$, defined as the probability that the instantaneous SNR $\eta$ falls below a given threshold level $\eta_{\text {th }}$ is obtained as

$$
\begin{aligned}
& P_{\text {out }}=\mathbb{P}\left(\eta \leq \eta_{\mathrm{th}}\right)=\mathbb{P}\left(\chi_{11} \chi_{12} \gamma_{s} \leq \eta_{\mathrm{th}}\right) \\
& =\mathbb{E}\left\{\mathbb{P}\left(\chi_{11} \leq \frac{\eta_{\mathrm{th}}}{\chi_{12} \gamma_{s}} \mid \chi_{12}\right)\right\}=1-\mathbb{E}\left\{\exp \left(-\frac{a}{\chi_{12}}\right)\right\} \\
& =1-\int_{0}^{\infty} \frac{1}{\bar{\chi}_{12}} \exp \left(-\frac{a}{x}-\frac{x}{\bar{\chi}_{12}}\right) d x,
\end{aligned}
$$

where $\mathbb{P}(A)$ stands for the probability of an event $A, \mathbb{E}(\cdot)$ denotes the expectation operator, and $a=\eta_{\mathrm{th}} /\left(\bar{\chi}_{11} \gamma_{s}\right)$. Using [19, Eq.(3-324.1)], it can be shown that the outage probability is obtained as

$$
P_{\text {out }}=1-\sqrt{\frac{4 \eta_{\mathrm{th}}}{\bar{\eta}}} K_{1}\left(\sqrt{\frac{4 \eta_{\mathrm{th}}}{\bar{\eta}}}\right),
$$

where $\bar{\eta}$ is the average SNR given as $\bar{\eta}=\bar{\chi}_{11} \bar{\chi}_{12} \gamma_{s}$, while $K_{1}(\cdot)$ is the modified Bessel function of first order.

The PDF of the instantaneous SNR $\eta$ is written as [20]

$$
p_{\eta}(x)=\frac{2}{\bar{\eta}} K_{0}\left(2 \sqrt{\frac{x}{\bar{\eta}}}\right),
$$

with $K_{0}(\cdot)$ being the modified Bessel function of zeroth order. Note that $\chi$ follows the same distribution as $\eta$. Using (4), we can express the ergodic capacity of the double Rayleigh channel with CSI at the transmitter as follows

$$
\begin{aligned}
\bar{C} & =\int_{0}^{\infty} \log _{2}\left(1+\gamma_{s} x\right) p_{\chi}(x) d x \\
& =\frac{\log _{2}(e)}{\gamma_{s} \bar{\chi}} G_{1,3}^{3,1}\left[\frac{1}{\gamma_{s} \bar{\chi}} \mid \begin{array}{l}
-1 \\
-1,-1,0
\end{array}\right],
\end{aligned}
$$

where $G_{p, q}^{m, n}(\cdot)$ is the Meijer G-function defined using a contour integral representation as [21]

$$
\begin{aligned}
& G_{p, q}^{m, n}\left(z \mid \begin{array}{l}
a_{1}, \ldots, a_{p} \\
b_{1}, \ldots, b_{q}
\end{array}\right)=\frac{1}{j 2 \pi} \\
& \times \int_{\mathcal{C}} \frac{\prod_{i=1}^{m} \Gamma\left(b_{i}+s\right) \prod_{i=1}^{n} \Gamma\left(1-a_{i}-s\right)}{\prod_{i=n+1}^{p} \Gamma\left(a_{i}+s\right) \prod_{i=m+1}^{q} \Gamma\left(1-b_{i}-s\right)} z^{-s} d s
\end{aligned}
$$

\footnotetext{
${ }^{1}$ Henceforth, for ease of notation, we drop the time dependence.
}

where $\Gamma(\cdot)$ denotes the gamma function [22].

\section{HARQ-CC THROUGHPUT ANALYSIS}

In this section, we first explain the HARQ-CC scheme and how it operates. Then, we derive the a generic expression of the throughput of HARQ-CC without assuming a specific distribution of the fading. Afterwards, we conduct the analysis of the throughput of HARQ-CC in the case of single Rayleigh channel and double Rayleigh channel.

\section{A. System Model}

In HARQ-CC, the transmitter sends a data packet and waits for the receiver feedback. If the transmitter receives an NACK, it sends the same data packet again until a maximum of $M$ rounds is reached or a successful decoding occurs. The receiver combines all received versions of the same data packet and performs maximum likelihood decoding. As the number of HARQ rounds increases, the coding rate decreases. The lowest coding rate corresponds to the case where $M$ HARQ rounds are utilized for a given data packet. The capacity $C_{m}$ in bits/symbol of HARQ-CC after $m$ rounds is expressed as

$$
C_{m}=\frac{1}{m} \log _{2}\left(1+\gamma_{s} \sum_{i=1}^{m} \chi_{i}\right), m=1,2, \ldots, M
$$

where $\chi_{i}$ is the squared envelope of the channel gain during the $i$ th transmission round. The random variables $\chi_{i}(i=$ $1, \ldots, m)$ are independent identically distributed (i.i.d.) and follow the same distribution. In this section, we do not assume a specific distribution of the fading and we present generic analysis of HARQ-CC.

It is worth mentioning that the number of retransmissions can vary from one data packet to another depending on the channel conditions. If the channel conditions are good, one HARQ round could be sufficient for error-free decoding. In the case of bad channel conditions, $M$ HARQ rounds might be required to transmit successfully one data packet. Thus, the transmission rate needs to be defined in a proper way. Towards this aim, let us assume that the transmitted data packet contains $b$ information bits. In each HARQ round, $L$ symbols are transmitted. The transmission rate of the first HARQ round in bits per channel use is written as $R_{1}=b / L$. Note that the transmission rate varies depending on the number of HARQ rounds used to transmit a data packet. The transmission rate is equal to $R_{m}=R_{1} / m$ if $m$ HARQ rounds are used to transmit successfully one data packet. We denote by $Q_{n}$ the number of HARQ rounds required for an error-free transmission of the $n$th data packet. The throughput $\bar{R}$ for $N$ data packets is expressed as

$$
\bar{R}=\frac{N b}{L \sum_{n=1}^{N} Q_{n}}=\frac{R_{1}}{\frac{1}{N} \sum_{n=1}^{N} Q_{n}}=\frac{R_{1}}{\mathbb{E}\left(T_{r}\right)}
$$

with $\mathbb{E}\left(T_{r}\right)$ being the average number of transmissions per data packet. The term $T_{r}$ is a random variable that represents the total number of transmissions of a given data packet.

The expression in (8) is valid for the case where there is no delay constraint, i.e., $M \rightarrow \infty$. However, if $M$ is finite, then 
the transmission of a data packet may fail after $M$ rounds. This event is called an outage event. The communication system is in outage if the capacity $C_{M}$ is less than the rate $R_{M}$. Hence, an outage occurs if the accumulated mutual information after $M$ rounds is less than the rate $R_{1}$. The outage probability of HARQ-CC after $M$ rounds reads as

$$
P_{\text {out }}^{\mathrm{CC}, \mathrm{M}}\left(R_{1}\right)=\mathbb{P}\left\{\log _{2}\left(1+\gamma_{s} \sum_{i=1}^{M} \chi_{i}\right) \leq R_{1}\right\} .
$$

Note that for $M \rightarrow \infty$, the outage probability $P_{\text {out }}^{\mathrm{CC}, \mathrm{M}}\left(R_{1}\right)$ becomes equal to zero and the throughput $\bar{R}$ reduces to the expression provided in (8). However, for a finite value of $M$, the outage probability is nonzero and the throughput is determined as

$$
\bar{R}=\frac{R_{1}\left(1-P_{\text {out }}^{\mathrm{CC}, M}\left(R_{1}\right)\right)}{\mathbb{E}\left(T_{r}\right)} .
$$

A similar expression of the throughput has been provided in [23]. From (10), one can conclude that if we choose a large value of $R_{1}$, the outage probability after $M$ HARQ rounds is approximately one, and the throughput $\bar{R}$ in (10) tends to zero. In order to avoid such a situation, we have to maintain the outage probability under a certain threshold $\epsilon$ and choose the rate $R_{1}$ subject to this threshold $\epsilon$.

\section{B. Outage Analysis and Throughput}

The $\epsilon$-outage capacity $C_{\epsilon}^{M}$ after $M$ rounds is defined in [24] as the largest transmission rate $R_{1}$ such that the outage probability is less than or equal to $\epsilon$, i.e., $P_{\text {out }}^{\mathrm{CC}, \mathrm{M}}\left(R_{1}\right) \leq \epsilon$. The $\epsilon$-outage capacity $C_{\epsilon}^{M}$ is obtained by solving the equation

$$
P_{\text {out }}^{\mathrm{CC}, \mathrm{M}}\left(C_{\epsilon}^{M}\right)=\epsilon .
$$

We set the rate of the first block as $R_{1}=C_{\epsilon}^{M}$. In this way, we guarantee that the probability of an outage is equal to $\epsilon$ after $M$ HARQ rounds. A fixed outage probability is guaranteed independently of the SNR level. The throughput of HARQ$\mathrm{CC}$ is obtained using (10) as

$$
C_{\epsilon}^{\mathrm{CC}, \mathrm{M}}=\frac{C_{\epsilon}^{M}\left(1-P_{\mathrm{out}}^{\mathrm{CC}, M}\left(C_{\epsilon}^{M}\right)\right)}{\mathbb{E}\left(T_{r}\right)}=\frac{C_{\epsilon}^{M}(1-\epsilon)}{\mathbb{E}\left(T_{r}\right)} .
$$

It can be shown that the average number of transmissions $\mathbb{E}\left(T_{r}\right)$ with a maximum number of rounds $M$ is given as

$$
\mathbb{E}\left(T_{r}\right)=1+\sum_{m=1}^{M-1} P\left(F^{1}, \ldots, F^{m}\right),
$$

where $F^{m}$ denotes the event decoding failure after $m$ HARQ rounds. The probability $P\left(F^{1}, \ldots, F^{m}\right)$ of a transmission failure after $m$ rounds decreases as $m$ increases. This probability is equal to the outage probability $P_{\mathrm{out}}^{\mathrm{CC}, \mathrm{m}}\left(R_{1}\right)$ after $m$ HARQ rounds. Thus, using (9), it follows

$$
\begin{aligned}
& P\left(F^{1}, \ldots, F^{m}\right)=P_{\text {out }}^{\mathrm{CC}, \mathrm{m}}\left(R_{1}\right)=P_{\text {out }}^{\mathrm{CC}, \mathrm{m}}\left(C_{\epsilon}^{M}\right) \\
& =\mathbb{P}\left\{\log _{2}\left(1+\gamma_{s} \sum_{i=1}^{m} \chi_{i}\right) \leq C_{\epsilon}^{M}\right\} \\
& =\mathbb{P}\left\{\sum_{i=1}^{m} \chi_{i} \leq \frac{2^{C_{\epsilon}^{M}}-1}{\gamma_{s}}\right\}=F_{\chi_{m}^{s}}\left(\frac{2^{C_{\epsilon}^{M}}-1}{\gamma_{s}}\right),
\end{aligned}
$$

where $F_{\chi_{m}^{s}}(x)$ is the cumulative distribution function (CDF) of $\chi_{m}^{s}=\sum_{i=1}^{m} \chi_{i}$. Note that the expression in (14) is valid for any distribution of $\chi_{i}$.

To determine the probability $P\left(F^{1}, \ldots, F^{m}\right)$ of a transmission failure after $m$ rounds, we need an expression of the $\epsilon$-outage capacity $C_{\epsilon}^{M}$ of HARQ-CC, which can be obtained by solving (11)

$\epsilon=\mathbb{P}\left\{\log _{2}\left(1+\gamma_{s} \sum_{i=1}^{M} \chi_{i}\right) \leq C_{\epsilon}^{M}\right\}=F_{\chi_{M}^{s}}\left(\frac{2^{C_{\epsilon}^{M}}-1}{\gamma_{s}}\right)$.

It follows that the $\epsilon$-outage capacity $C_{\epsilon}^{M}$ is

$$
C_{\epsilon}^{M}=\log _{2}\left(1+\gamma_{s} F_{\chi_{M}^{s}}^{-1}(\epsilon)\right),
$$

where $F_{\chi_{M}^{s}}^{-1}(\cdot)$ is the inverse of the $\operatorname{CDF} F_{\chi_{M}^{s}}(\cdot)$.

Using (13)-(16), the average number of transmissions $\mathbb{E}\left(T_{r}\right)$ is obtained as

$$
\mathbb{E}\left(T_{r}\right)=1+\sum_{m=1}^{M-1} F_{\chi_{m}^{s}}\left(\frac{2_{\epsilon}^{C_{\epsilon}^{M}}-1}{\gamma_{s}}\right)=1+\sum_{m=1}^{M-1} F_{\chi_{m}^{s}}\left(F_{\chi_{M}^{s}}^{-1}(\epsilon)\right) .
$$

Note that the average number of transmissions $\mathbb{E}\left(T_{r}\right)$ is independent of the SNR. In fact, the transmission rate $C_{\epsilon}^{M}$ per HARQ round depends on the SNR. As the SNR increases, the transmission rate increases as well. In contrast to systems that have a fixed transmission rate, we have a variable rate, which is adjusted to the SNR such that a fixed target outage probability $\epsilon$ is achieved. This setting is in line with contemporary communication systems, where a certain quality of service should be guaranteed to users, independently of the channel conditions. For a given value of $\epsilon$, the average number of transmissions $\mathbb{E}\left(T_{r}\right)$ is only a function of the maximum number of rounds $M$. Hence, for fixed values of $M$ and $\epsilon$, the average number of transmissions $\mathbb{E}\left(T_{r}\right)$ is constant for all SNR values.

Substituting (16) and (17) in (12), we can obtain the throughput $C_{\epsilon}^{\mathrm{CC}, \mathrm{M}}$ of HARQ-CC as

$$
C_{\epsilon}^{\mathrm{CC}, \mathrm{M}}=\frac{\log _{2}\left(1+\gamma_{s} F_{\chi_{M}^{s}}^{-1}(\epsilon)\right)(1-\epsilon)}{1+\sum_{m=1}^{M-1} F_{\chi_{m}^{s}}\left(F_{\chi_{M}^{s}}^{-1}(\epsilon)\right)} .
$$

We define a fixed time diversity scheme as a communication scheme that retransmits the same packet over $M$ rounds without the use of acknowledgment indicating the success/failure of a packet transmission. Subsequently, this system is nonadaptive and has a fixed time diversity equal to $M$. The throughput of the fixed time diversity scheme is obtained as

$$
C_{\epsilon}^{\mathrm{No}-\mathrm{HARQ}, \mathrm{M}}=\frac{\log _{2}\left(1+\gamma_{s} F_{\chi_{M}^{s}}^{-1}(\epsilon)\right)(1-\epsilon)}{M} .
$$

\section{Throughput over Rayleigh and Double Rayleigh Fading Channels}

If the fading channel $\chi_{i}$ in each HARQ round follows a Rayleigh distribution, then $\chi_{m}^{s}=\sum_{i=1}^{m} \chi_{i}$ follows a chisquare distribution with $2 m$ degrees of freedom. Thus, the 
$\mathrm{CDF} F_{\chi_{m}^{s}}(x)$ of $\chi_{m}^{s}$ is obtained as [25]

$$
F_{\chi_{m}^{s}}(x)=1-\exp \left(-\frac{x}{\bar{\chi}}\right) \sum_{i=0}^{m-1} \frac{1}{i !}\left(\frac{x}{\bar{\chi}}\right)^{i} .
$$

Using [26, Eq. (06.06.03.0009.01)], [22, Eq. (6.5.3)], and the definition of the incomplete gamma function $\Gamma_{a}(\cdot)$ in [22, Eq. (6.5.1)], we can express the $\operatorname{CDF} F_{\chi_{m}^{s}}(x)$ as

$$
F_{\chi_{m}^{s}}(x)=\Gamma_{m}\left(\frac{x}{\bar{\chi}}\right) \text {. }
$$

Using (21) and (16), the $\epsilon$-outage capacity $C_{\epsilon}^{M}$ for the case of Rayleigh channel is expressed as

$$
C_{\epsilon}^{M}=\log _{2}\left(1+\bar{\chi} \gamma_{s} \Gamma_{M}^{-1}(\epsilon)\right)
$$

where $\Gamma_{a}^{-1}(\cdot)$ is the inverse of the incomplete gamma function $\Gamma_{a}(\cdot)$. The throughput $C_{\epsilon}^{\mathrm{CC}, \mathrm{M}}$ of HARQ-CC over Rayleigh channels can be derived using (21) and (18) as

$$
C_{\epsilon}^{\mathrm{CC}, \mathrm{M}}=\frac{\log _{2}\left(1+\bar{\chi} \gamma_{s} \Gamma_{M}^{-1}(\epsilon)\right)(1-\epsilon)}{1+\sum_{m=1}^{M-1} \Gamma_{m}\left(\Gamma_{M}^{-1}(\epsilon)\right)} .
$$

If the underlying fading channel $\chi_{i}$ in each HARQ round follows a double Rayleigh distribution there exists no closedform solution for the $\mathrm{CDF} F_{\chi_{m}^{s}}(x)$ of $\chi_{m}^{s}=\sum_{i=1}^{m} \chi_{i}$. Therefore, we derive in Appendix $A$ an approximate solution for the $\operatorname{CDF} F_{\chi_{m}^{s}}(x)$, which is obtained as

$$
F_{\chi_{m}^{s}}(x) \approx \Gamma_{\alpha_{\chi_{m}^{s}}+1}\left(\frac{x}{\beta_{\chi_{m}^{s}}}\right)
$$

with $\Gamma_{a}(\cdot)$ being the incomplete gamma function [22, Eq. (6.5.1)]. The expressions of the quantities $\alpha_{\chi_{m}^{s}}$ and $\beta_{\chi_{m}^{s}}$ are derived in Appendix A.

Using (24) and (16), the $\epsilon$-outage capacity $C_{\epsilon}^{M}$ for the case of double Rayleigh channel is approximated as

$$
C_{\epsilon}^{M} \approx \log _{2}\left(1+\beta_{\chi_{M}^{s}} \gamma_{s} \Gamma_{\alpha_{\chi_{M}^{s}}^{s}+1}^{-1}(\epsilon)\right)
$$

where $\Gamma_{a}^{-1}(\cdot)$ is the inverse of the incomplete gamma function $\Gamma_{a}(\cdot)$. The throughput $C_{\epsilon}^{\mathrm{CC}, \mathrm{M}}$ of HARQ-CC over double Rayleigh channels can be derived using (24) and (18) as

$$
C_{\epsilon}^{\mathrm{CC}, \mathrm{M}} \approx \frac{\log _{2}\left(1+\beta_{\chi_{M}^{s}} \gamma_{s} \Gamma_{\alpha_{\chi_{M}^{s}+1}^{-1}}^{-1}\right)(1-\epsilon)}{1+\sum_{m=1}^{M-1} \Gamma_{\alpha_{\chi_{m}^{s}}+1}\left(\Gamma_{\alpha_{\chi_{M}^{s}}^{-1}+1}(\epsilon)\right)}
$$

The throughput of the fixed time diversity scheme is obtained as

$$
C_{\epsilon}^{\mathrm{No}-\mathrm{HARQ}, \mathrm{M}} \approx \frac{\log _{2}\left(1+\beta_{\chi_{M}^{s}} \gamma_{s} \Gamma_{\alpha_{\chi_{M}^{s}}^{-1}+1}^{-}(\epsilon)\right)(1-\epsilon)}{M} .
$$

\section{Optimal Transmission Rate Per Round In HARQ-CC}

In this section, we aim to maximize the throughput $C_{\epsilon}^{\mathrm{CC}, \mathrm{M}}$ by optimizing the value of the rate $R_{1}$ per HARQ round. In [27], it has been demonstrated that using a rate $R_{1}$ smaller then $C_{\epsilon}^{M}$ allows increasing the throughput compared to the case when the value of $R_{1}$ equals $C_{\epsilon}^{M}$. For instance, by setting $R_{1}$ to $0.7 C_{\epsilon}^{M}$, the throughput $C_{\epsilon}^{\mathrm{CC}, \mathrm{M}}$ increases if $M$ increases for low and high SNR values. We formulate the optimization problem as follow. First, we express the rate $R_{1}$ as a fraction of $C_{\epsilon}^{M}$, i.e., $R_{1}=\alpha C_{\epsilon}^{M}$, where $\alpha \in[0,1]$. Note that $R_{1} \leq C_{\epsilon}^{M}$ which ensures that the system outage probability is less then $\epsilon$. The optimization problem is written as follows

$$
\underset{\alpha}{\operatorname{maximize}} C_{\epsilon}^{\mathrm{CC}, \mathrm{M}}(\alpha)=\frac{\alpha C_{\epsilon}^{M}(1-\epsilon)}{1+\sum_{m=1}^{M-1} \Gamma_{\alpha_{\chi_{m}^{s}}+1}\left(\frac{2^{\alpha C_{\epsilon}^{M}}-1}{\beta_{\chi_{m}^{s}} \gamma_{s}}\right)}
$$

subject to $0<\alpha<1$.

Note that the optimization problem can only be solved numerically. The optimal value of $\alpha$, which maximizes the throughput, will be denoted by $\alpha^{*}$. From our investigation, we notice that the value of $\alpha^{*}$ varies depending on the maximum number of retransmissions $M$ and the SNR value.

\section{HARQ-CC WITH IMPERFECT FEEDBACK}

In this section, we analyze the performance of HARQCC over double Rayleigh channels with imperfect feedback channels. In this realistic case of imperfect acknowledgements, the received ACK/NACK does not always match the transmitted acknowledgement. We recall that an ACK sent from the receiver to the transmitter indicates that the packet has been received correctly at the receiver. Once an ACK message is received, the transmitter moves on to the next data packet. A NACK message indicate that the packet has not been successfully received. When the transmitter receives a NACK, it sends the same packet in the next transmission rounds until it receives an ACK or maximum number of retransmissions $M$ is reached.

We can define two possible error scenarios related to the imperfect feedback channel. In the first scenario, an ACK is sent by the receiver, and the transmitter detects it as a NACK due to the imperfect feedback channel. We use the notation $\mathrm{ACK} \rightarrow \mathrm{NACK}$ to refer to this kind of error. Note that if an ACK $\rightarrow$ NACK error occurs, the current data packet is retransmitted instead of moving on to the next packet. The retransmission process is repeated until the ACK is correctly received. In the second error scenario, a NACK $\rightarrow$ ACK error occurs. This setup is more critical because the transmitter automatically sends the next data packet even though the current one has been erroneously received. In this case, the current data packet is lost.

In the following, we derive an expression for the probability that a packet is lost. A packet is lost after the first round, if a reception failure occurs at the first round and a NACK $\rightarrow$ ACK error happens. This event occurs with a probability $P\left(F^{1}\right) \epsilon_{f b}$, where $\epsilon_{f b}$ is the feedback error probability and $P\left(F^{1}\right)$ the 
probability of transmission failure after the first HARQ round. A packet is lost at round $j(1 \leq j \leq M-1)$, if a reception failure occurs after $j$ rounds and a NACK $\rightarrow$ ACK error takes place in the $j$ th round, whereas the first $j$ NACKs are received correctly. This event happens with probability $P\left(F^{1}, \ldots, F^{j}\right)\left(1-\epsilon_{f b}\right)^{j-1} \epsilon_{f b}$. A packet is lost in the $M$ th round, if a reception failure after $M$ rounds occurs and correct NACKs are received for the first $M-1$ th rounds. The packet is lost regardless whether a NACK or an ACK is detected at the receiver. The probability of a packet loss in round $M$ is written as $P\left(F^{1}, \ldots, F^{M}\right)\left(1-\epsilon_{f b}\right)^{M-1}$. Since the events of loosing a packet in round $1,2, \ldots, M$ are mutually exclusive, the probability of packet loss is the sum of the probability of these events and is expressed as

$$
\begin{aligned}
& \xi_{M}=P\left(F^{1}\right) \epsilon_{f b}+P\left(F^{1}, F^{2}\right)\left(1-\epsilon_{f b}\right) \epsilon_{f b}+\cdots \\
& +P\left(F^{1}, \ldots, F^{j}\right) \cdot\left(1-\epsilon_{f b}\right)^{j-1} \epsilon_{f b}+\cdots+P\left(F^{1}, \ldots, F^{M-1}\right) \\
& \cdot\left(1-\epsilon_{f b}\right)^{M-2} \epsilon_{f b}+P\left(F^{1}, \ldots, F^{M}\right)\left(1-\epsilon_{f b}\right)^{M-1} .
\end{aligned}
$$

In the case of an imperfect feedback channel, the transmission rate is expressed as

$$
C_{\epsilon}^{\mathrm{Imper}, \mathrm{M}}=\frac{C_{\epsilon}^{M}\left(1-\xi_{M}\right)}{\mathbb{E}\left(T_{r, I}\right)},
$$

where $\mathbb{E}\left(T_{r, I}\right)$ is the average number of transmissions with imperfect feedback. The expression of $\mathbb{E}\left(T_{r, I}\right)$ is derived as

$$
\begin{aligned}
& \mathbb{E}\left(T_{r, I}\right)=\sum_{m=1}^{M-1} m\left(P\left(F^{1}, \ldots, F^{m}\right)\left(1-\epsilon_{f b}\right)^{m-1} \epsilon_{f b}\right. \\
& +\left[1-P\left(F^{1}\right)\right]\left(1-\epsilon_{f b}\right) \epsilon_{f b}^{m-1}+\sum_{j=2}^{m}\left(1-\epsilon_{f b}\right)^{j} \epsilon_{f b}^{m-j} \\
& \left.\cdot\left[P\left(F^{1}, \ldots, F^{j-1}\right)-P\left(F^{1}, \ldots, F^{j}\right)\right]\right)+M\left(\left(1-\epsilon_{f b}\right)^{M-1}\right. \\
& \cdot P\left(F^{1}, \ldots, F^{M-1}\right)+\left[1-P\left(F^{1}\right)\right] \epsilon_{f b}^{M-1}+\sum_{j=2}^{M-1} \epsilon_{f b}^{M-j} \\
& \left.\cdot\left(1-\epsilon_{f b}\right)^{j-1}\left[P\left(F^{1}, \ldots, F^{j-1}\right)-P\left(F^{1}, \ldots, F^{j}\right)\right]\right) .
\end{aligned}
$$

Proof: See Appendix B.

\section{Vi. Delay Analysis with Perfect and Imperfect FEEDBACK CHANNELS}

In this section, we analyse the communication system delay with perfect and imperfect feedback channels. In order to control the flow of the packets in the network, we consider that the source is equipped with a buffer to store the packets before transmission. We assume Poisson arriving packets at the buffer with arrival rate $\lambda$. We study in this section the average waiting time for a data packet and the average sojourn time in the buffer. Analytical expressions are derived for these terms - first for the case of perfect feedback channels and then for imperfect feedback channels.

The average waiting time $W$ for a data packet is the time spent by the packet in the buffer of the transmitter starting from the arrival of the packet until the start of the first HARQ round associated with that packet. The average waiting time $W$ is determined using the Pollaczek-Khinchin equation [28]

$$
W=\frac{\lambda \mathbb{E}\left(T_{r}^{2}\right) T_{F}^{2}}{2(1-\rho)}+\frac{T_{F}}{2},
$$

where $T_{F}$ is the frame duration and $\rho$ is a parameter that should satisfy the following stability condition

$$
\rho=\lambda \mathbb{E}\left(T_{r}\right) T_{F}<1
$$

The term $\mathbb{E}\left(T_{r}^{2}\right)$ refers to the second-order moment of the number of transmissions $T_{r}$. With a perfect feedback channel and an optimal transmission rate $R_{1}=\alpha^{*} C_{\epsilon}^{M}$, the expression of the second-order moment of the number of transmissions is derived as [29]

$$
\mathbb{E}\left(T_{r}^{2}\right)=1+\sum_{m=1}^{M-1}(2 m+1) P\left(F^{1}, \ldots, F^{m}\right),
$$

where the term $P\left(F^{1}, \ldots, F^{m}\right)$ can be approximated using (14) and (24) as

$$
P\left(F^{1}, \ldots, F^{m}\right) \approx \Gamma_{\alpha_{\chi_{m}^{s}}+1}\left(\frac{2^{R_{1}}-1}{\beta_{\chi_{m}^{s}} \gamma_{s}}\right) .
$$

The average sojourn time $T_{\text {soj }}$ in the buffer is the average time elapsed from the packet arrival until its successful reception at the receiver. The average sojourn time is written as the sum of two terms: (i) the average waiting time $W$ and (ii) the time for successfully transmitting a data packet [30], i.e.,

$$
T_{\text {soj }}=W+\mathbb{E}\left(T_{S}\right) T_{F} .
$$

$\mathbb{E}\left(T_{S}\right)$ denotes the average number of transmissions required to successfully transmit a data packet. It can be shown that $\mathbb{E}\left(T_{S}\right)$ can be expressed in terms of $\mathbb{E}\left(T_{r}\right)$ as

$$
\mathbb{E}\left(T_{S}\right)=\mathbb{E}\left(T_{r}\right)-M P\left(F^{1}, \ldots, F^{M}\right) .
$$

\section{Proof: See Appendix C.}

So far, we have provided expressions for the average waiting time $W$ and the sojourn time $T_{\text {soj }}$ for the case of perfect feedback channels. For the case of imperfect feedback channels, the average waiting time is denoted by $W_{I}$, while the sojourn time is referred to as $T_{\mathrm{soj}, I}$. The average waiting time $W_{I}$ is expressed as

$$
W_{I}=\frac{\lambda \mathbb{E}\left(T_{r, I}^{2}\right) T_{F}^{2}}{2\left(1-\lambda \mathbb{E}\left(T_{r, I}\right) T_{F}\right)}+\frac{T_{F}}{2},
$$

where $\mathbb{E}\left(T_{r, I}\right)$ is the average number of transmissions with imperfect feedback and its expression is provided in (31). The term $\mathbb{E}\left(T_{r, I}^{2}\right)$ stands for the second-order moment of the number of transmissions with imperfect feedback, which can 
be expressed as

$$
\begin{aligned}
& \mathbb{E}\left(T_{r, I}^{2}\right)=\sum_{m=1}^{M-1} m^{2}\left(P\left(F^{1}, \ldots, F^{m}\right)\left(1-\epsilon_{f b}\right)^{m-1} \epsilon_{f b}\right. \\
& +\left[1-P\left(F^{1}\right)\right]\left(1-\epsilon_{f b}\right) \epsilon_{f b}^{m-1}+\sum_{j=2}^{m}\left(1-\epsilon_{f b}\right)^{j} \epsilon_{f b}^{m-j} \\
& \left.\cdot\left[P\left(F^{1}, \ldots, F^{j-1}\right)-P\left(F^{1}, \ldots, F^{j}\right)\right]\right)+M^{2}\left(\left(1-\epsilon_{f b}\right)^{M-1}\right. \\
& \cdot P\left(F^{1}, \ldots, F^{M-1}\right)+\left[1-P\left(F^{1}\right)\right] \epsilon_{f b}^{M-1}+\sum_{j=2}^{M-1} \epsilon_{f b}^{M-j} \\
& \left.\cdot\left(1-\epsilon_{f b}\right)^{j-1}\left[P\left(F^{1}, \ldots, F^{j-1}\right)-P\left(F^{1}, \ldots, F^{j}\right)\right]\right)
\end{aligned}
$$

The sojourn time $T_{\mathrm{soj}, I}$ with imperfect feedback channels is obtained as

$$
T_{\mathrm{soj}, I}=W_{I}+\mathbb{E}\left(T_{S, I}\right) T_{F},
$$

where $\mathbb{E}\left(T_{S, I}\right)$ refers to the average number of successful transmissions with imperfect feedback. The expression of $\mathbb{E}\left(T_{S, I}\right)$ can be derived as

$$
\begin{aligned}
& \mathbb{E}\left(T_{S, I}\right)=1 P\left(S^{1}\right)\left(1-\epsilon_{f b}\right)+\sum_{j=2}^{M} j P\left(F^{1}, \ldots, F^{j-1}, S^{j}\right) \\
& \cdot\left(1-\epsilon_{f b}\right)^{j}+\sum_{m=2}^{M} \sum_{j=1}^{m-1} j P\left(F^{1}, \ldots, F^{j-1}, S^{j}\right)\left(1-\epsilon_{f b}\right)^{j} \epsilon_{f b}^{m-j}
\end{aligned}
$$

where $P\left(S^{1}\right)$ and $P\left(F^{1}, \ldots, F^{j-1}, S^{j}\right)$ are the probabilities of successful transmission after the first and the $j$ th round, respectively. Note that $P\left(S^{1}\right)=1-$ $P\left(F^{1}\right)$, while $P\left(F^{1}, \ldots, F^{j-1}, S^{j}\right)=P\left(F^{1}, \ldots, F^{j-1}\right)-$ $P\left(F^{1}, \ldots, F^{j-1}, F^{j}\right)$. The proof of (41) is not provided due to space limitation.

\section{ENERGY EFFICIENCY}

This section is dedicate for studying the energy efficiency $\eta_{E E}$ of HARQ-CC over double Rayleigh channels. The energy efficiency indicates how much energy is spent in average for each successfully received bit and is measured in bits per Joule. The energy efficiency of a communication system is defined as the ratio between the throughput and the average consumed power and is given by [31], [32]

$$
\eta_{E E}=\frac{C_{\epsilon}^{M}(1-\epsilon)}{\overline{\mathcal{P}}},
$$

where $C_{\epsilon}^{M}$ is the $\epsilon$-outage capacity. The term $\overline{\mathcal{P}}$ denotes the average power consumption, which is expressed as the product of two terms: (i) the transmission power $\mathcal{P}$ per HARQ round and (ii) the average number of transmissions $\mathbb{E}\left(T_{r}\right)$, i.e.,

$$
\overline{\mathcal{P}}=\mathcal{P} \mathbb{E}\left(T_{r}\right)
$$

Using (42) and (43) in combination with (12), the energy efficiency $\eta_{E E}$ reads as

$$
\eta_{E E}=\frac{C_{\epsilon}^{M}(1-\epsilon)}{\mathcal{P} \mathbb{E}\left(T_{r}\right)}=\frac{C_{\epsilon}^{\mathrm{CC}, M}}{\mathcal{P}} .
$$

We also investigate the tradeoff between the energy efficiency $\eta_{E E}$ and the throughput $C_{\epsilon}^{\mathrm{CC}, M}$ of HARQ-CC. Our study reveals that the energy efficiency and the throughput cannot be maximized jointly, which shows that there is a tradeoff between these two quantities. This tradeoff is investigated in more detail in Section VIII.

\section{NUMERICAL RESULTS}

\section{A. Throughput and Average Number of Transmissions}

In this section, the analytical expressions for the average number of transmissions $\mathbb{E}\left(T_{r}\right)$ and the throughput $C_{\epsilon}^{\mathrm{CC}, M}$ of HARQ-CC are numerically evaluated and illustrated for the case of perfect feedback channels. The impact of using an optimal rate per round $R_{1}$ on the throughput $C_{\epsilon}^{\mathrm{CC}, M}$ is highlighted and discussed.

In our analysis, since there is no closed-form expression for the CDF $F_{\chi_{M}^{s}}(x)$ of the sum of double Rayleigh RVs $\chi_{M}^{s}=$ $\sum_{i=1}^{M} \chi_{i}$, we approximate this $\mathrm{CDF} F_{\chi_{M}^{s}}(x)$ by the CDF of a gamma distribution [see (24)]. To illustrate the accuracy of this approximation, we provide in Fig. 1 the exact CDF of $\chi_{M}^{s}$ obtained using Monte Carlo simulations together with the gamma approximation of the CDF of $\chi_{M}^{s}$. From Fig. 1, it can be concluded that the gamma approximation have a good accuracy for different values of the SNR and $M$. Moreover, as the value of $M$ increases the gamma approximation becomes more accurate.

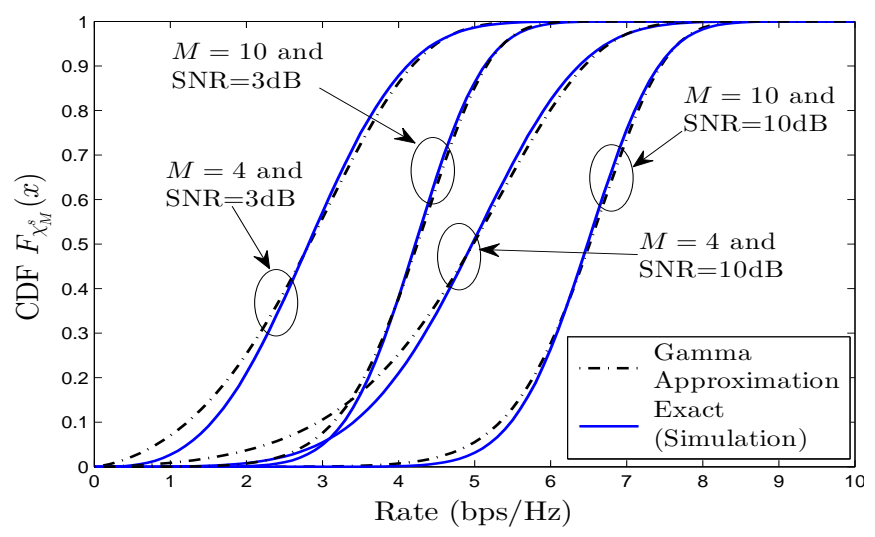

Fig. 1. Gamma approximation and exact $\mathrm{CDF} F_{\chi_{M}^{s}}(x)$ of the sum of double Rayleigh RVs $\chi_{M}^{s}=\sum_{i=1}^{M} \chi_{i}$ for $M=4$ and 10 and for SNR equal to 3 and $10 \mathrm{~dB}$.

In Fig. 2, we plot the throughput $C_{\epsilon}^{\mathrm{CC}, \mathrm{M}}$ of HARQ-CC for $M=6,9$ and 15 ; while the transmission rate per HARQ round $R_{1}$ is set to $C_{\epsilon}^{M}$ and $\alpha^{*} C_{\epsilon}^{M}$. By setting the transmission rate per HARQ round to $R_{1}=C_{\epsilon}^{M}$, we maintain the outage probability below $\epsilon=0.01$ after $M$ transmission rounds. However, this choice results in degrading the throughput. If we reduce the rate per round $R_{1}$ from $C_{\epsilon}^{M}$ to $\alpha^{*} C_{\epsilon}^{M}$, the throughput $C_{\epsilon}^{\mathrm{CC}, \mathrm{M}}$ is improved ${ }^{2}$ as confirmed in Fig. 3. In fact, $C_{\epsilon}^{\mathrm{CC}, \mathrm{M}}=R_{1}(1-\epsilon) / \mathbb{E}\left(T_{r}\right)$. It follows that the throughput $C_{\epsilon}^{\mathrm{CC}, \mathrm{M}}$ increases if $R_{1}$ increases or if the average number of transmissions $\mathbb{E}\left(T_{r}\right)$ decreases. On the other hand, if we

\footnotetext{
${ }^{2}$ Note that $\alpha^{*} \in[0,1]$, thus $\alpha^{*} C_{\epsilon}^{M} \leq C_{\epsilon}^{M}$.
} 
increase the rate $R_{1}$, the average number of transmissions $\mathbb{E}\left(T_{r}\right)$ increases as it is illustrated in Fig. 4. Note that $\alpha^{*} C_{\epsilon}^{M}$ is the optimal value of the rate $R_{1}$ which maximizes the throughput $C_{\epsilon}^{\mathrm{CC}, M}$. In Fig. 2, the throughput $C_{\epsilon}^{\mathrm{CC}, \mathrm{M}}$ increases

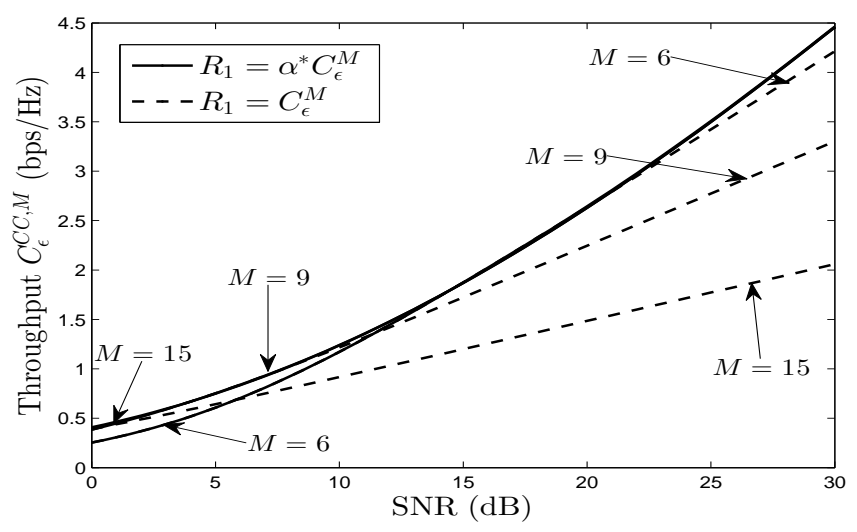

Fig. 2. Impact of the transmission rate $R_{1}$ per round on the throughput $C_{\epsilon}^{\mathrm{CC}, \mathrm{M}}$ for various values of $M$.

if the rate $R_{1}$ is reduced from $C_{\epsilon}^{M}$ to $\alpha^{*} C_{\epsilon}^{M}$. For instance, for $M=15$ and an SNR of $30 \mathrm{~dB}$, the throughput $C_{\epsilon}^{\mathrm{CC}, \mathrm{M}}$ increases from $2 \mathrm{bps} / \mathrm{Hz}$ to $4.5 \mathrm{bps} / \mathrm{Hz}$ if an optimal rate $\left(R_{1}=\alpha^{*} C_{\epsilon}^{M}\right)$ is used instead of a full rate $\left(R_{1}=C_{\epsilon}^{M}\right)$.

In Fig. 3, we illustrate the impact of the rate $R_{1}$ on the throughput $C_{\epsilon}^{\mathrm{CC}, \mathrm{M}}$. In this figure, the SNR equals $25 \mathrm{~dB}$, while the rate per round $R_{1}$ is set to $R_{1}=\alpha C_{\epsilon}^{M}$, with $\alpha$ being a constant within the interval $[0,1]$. From Fig. 3 , it can be seen that the rate $R_{1}$ which maximizes the throughput is less than $C_{\epsilon}^{M}$ since the optimal value of $\alpha^{*}<1$. The optimal value of $\alpha$ varies depending on the value of $M$. From Fig. 3, we can obtain the optimal values of $\alpha$ for an SNR of $25 \mathrm{~dB}$ as $0.61,0.72$ and 0.88 for $M$ equal to 15,9 and 6, respectively. Hence, it is possible to increase the throughput by using a rate per round $R_{1}$ smaller than $C_{\epsilon}^{M}$. Note that $C_{\epsilon}^{M}$ is the rate that achieves the target outage $\epsilon$ and that the rate $R_{1}$ that maximizes the throughput $C_{\epsilon}^{\mathrm{CC}, \mathrm{M}}$ leads to lower outage than the target outage $\epsilon$.

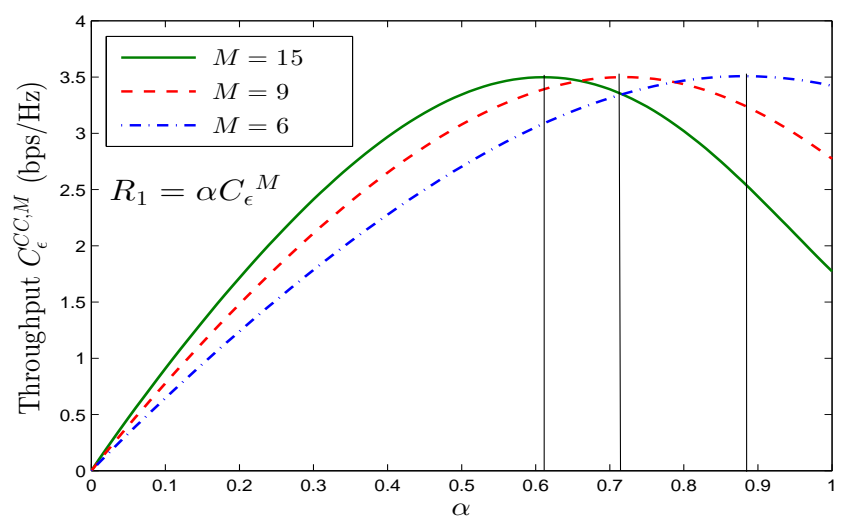

Fig. 3. The impact of the rate $R_{1}$ on the throughput $C_{\epsilon}^{\mathrm{CC}, \mathrm{M}}$ for different values of $M$ and an SNR of $25 \mathrm{~dB}$.

Fig. 4 illustrates the average number of transmissions $\mathbb{E}\left(T_{r}\right)$ versus the SNR for different values of $M$. It can be seen that
$\mathbb{E}\left(T_{r}\right)$ decreases if the rate $R_{1}$ per round is reduced from $C_{\epsilon}^{M}$ to $\alpha^{*} C_{\epsilon}^{M}$. If the rate $R_{1}$ is set to $R_{1}=C_{\epsilon}^{M}$, then $\mathbb{E}\left(T_{r}\right)$ is constant w.r.t. the SNR and increases with $M$. If the rate $R_{1}$ per round equals to the optimal rate, i.e., $R_{1}=\alpha^{*} C_{\epsilon}^{M}$, a significant reduction of the quantity $\mathbb{E}\left(T_{r}\right)$ is observed, especially for large values of $M$ at high SNR. Note that the decrease of $\mathbb{E}\left(T_{r}\right)$ results in an increase of the throughput $C_{\epsilon}^{\mathrm{CC}, \mathrm{M}}$. The impact of the rate $R_{1}$ optimization on the average number of transmissions $\mathbb{E}\left(T_{r}\right)$ confirms the results obtained in Fig. 2, where the use of an optimal rate $R_{1}$ per round increases the throughput $C_{\epsilon}^{\mathrm{CC}, \mathrm{M}}$ especially at high SNR.

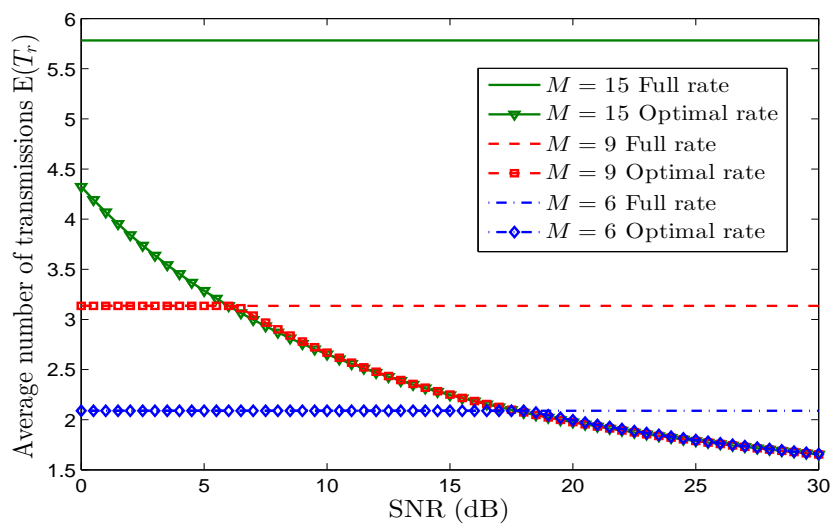

Fig. 4. The average number of transmissions $\mathbb{E}\left(T_{r}\right)$ for different values of the transmission rate $R_{1}$ per round for $\epsilon=0.01$.

In Fig. 5, we compare the throughput $C_{\epsilon}^{\mathrm{CC}, \mathrm{M}}$ of HARQ$\mathrm{CC}$ achieved by using the optimal rate $R_{1}=\alpha^{*} C_{\epsilon}^{M}$ and the throughput $C_{\epsilon}^{\mathrm{No}-\mathrm{HARQ}, \mathrm{M}}$ of a fixed time diversity scheme. The advantage of using HARQ can be clearly seen from Fig. 5. For instance, for $M=9$, a capacity gain of 3.64 $\mathrm{bps} / \mathrm{Hz}$ at an SNR of $30 \mathrm{~dB}$ is achieved by using HARQ-CC compared to the fixed time diversity scheme. For the latter scheme, the same packet is retransmitted over $M$ rounds due to the absence of feedback from the receiver. The expression of the throughput $C_{\epsilon}^{\mathrm{No}-\mathrm{HARQ}, \mathrm{M}}$ for the fixed time diversity scheme is provided in (27). A system that does not use HARQ suffers from a low throughput, especially at high SNR regime. In fact, for high SNR values, few transmission rounds are enough to transmit successfully a data packet, while the fixed time diversity scheme uses $M$ retransmissions. The HARQ technique adapts implicitly the number of transmissions to the channel conditions which improves the throughput considerably especially at high SNR, while the outage probability is maintained below a threshold level $\epsilon=0.01$.

In Fig. 6, we illustrate the throughput $C_{\epsilon}^{\mathrm{CC}, \mathrm{M}}$ of HARQ$\mathrm{CC}$ over both Rayleigh and double Rayleigh channels. These results have been obtained by setting the rate per round $R_{1}=C_{\epsilon}^{M}{ }^{3}$ The analytical expression of the throughput over Rayleigh channels is provided in (23), while the throughput over double Rayleigh channels is given by (26). From Fig. 6, it can be noticed that the double Rayleigh channel exhibits more sever fading and results in the degradation of the throughput

${ }^{3}$ Note that the expression of $C_{\epsilon}^{M}$ for the Rayleigh channel and the double Rayleigh channel are given by (22) and (25), respectively. 


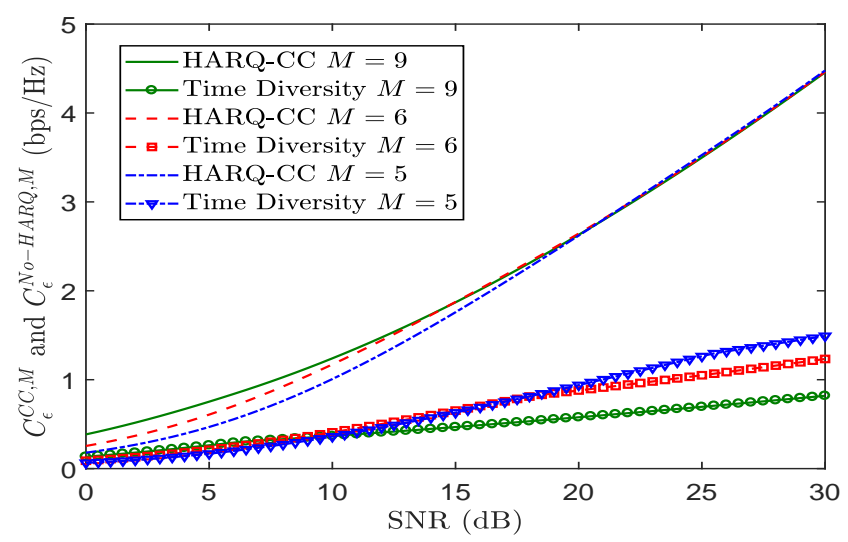

Fig. 5. Throughput $C_{\epsilon}^{\mathrm{CC}, \mathrm{M}}$ of HARQ-CC in comparison to the throughput $C_{\epsilon}^{\mathrm{No}-\mathrm{HARQ}, \mathrm{M}}$ of the fixed time diversity scheme for $\epsilon=0.01$.

significantly. Moreover, for the Rayleigh channel even without optimizing the rate per round $R_{1}$, the throughput increases as $M$ increases. This is as opposed to the double Rayleigh channel where the use of a rate $R_{1}=C_{\epsilon}^{M}$ leads to the decrease of the throughput as $M$ increases especially at high SNR [see Fig. 2]. By exploring the optimal value of $\alpha^{*}$ for the Rayleigh channel case, it turns out that $\alpha^{*}$ is within the interval $[0.95,1]$ which implies that the use of $\alpha=1$, i.e. $R_{1}=C_{\epsilon}^{M}$, results in a near optimal throughput ${ }^{4}$. While for the double Rayleigh case, the optimal value of $\alpha^{*}$ can reach 0.6 for $M=15$ which implies that we have to reduce the rate $R_{1}=C_{\epsilon}^{M}$ by $40 \%$ to maximize the throughput. It can be concluded that it is more critical to perform the optimization of the rate $R_{1}$ in the case of double Rayleigh channel to maximize the throughput $C_{\epsilon}^{\mathrm{CC}, \mathrm{M}}$ of HARQ-CC.

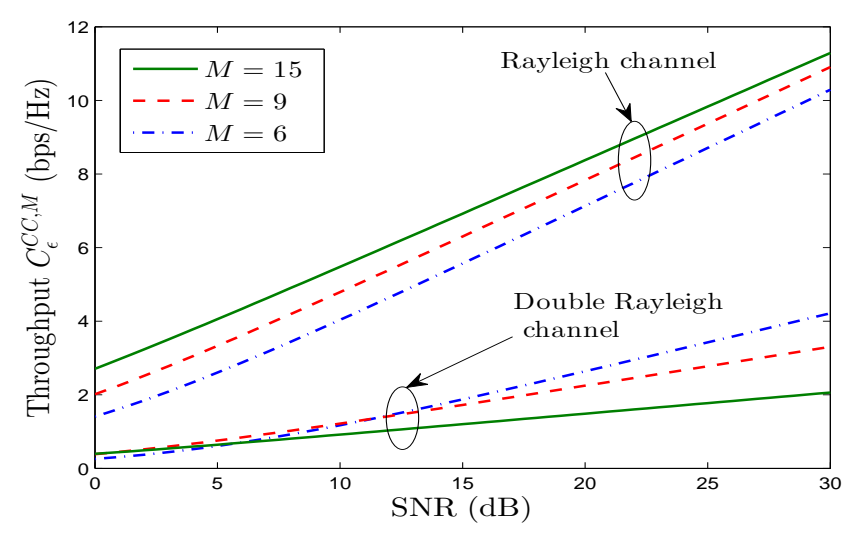

Fig. 6. Throughput $C_{\epsilon}^{\mathrm{CC}, \mathrm{M}}$ of HARQ-CC over Rayleigh and double Rayleigh channels for $R_{1}=C_{\epsilon}^{M}$.

In Fig. 7, we compare the throughput $C_{\epsilon}^{\mathrm{CC}, \mathrm{M}}$ of HARQ-CC to the throughput $C_{\epsilon}^{\mathrm{IR}, \mathrm{M}}$ of HARQ-IR over double Rayleigh channels. For the HARQ-CC scheme, the rate $R_{1}$ is optimized, i.e. $R_{1}=\alpha^{*} C_{\epsilon}^{M}$, while for the HARQ-IR scheme the rate is not optimized and set to $R_{1}=C_{\epsilon}^{M}$. The analytical expression of $C_{\epsilon}^{M}$ for the HARQ-IR scheme is provided in

\footnotetext{
${ }^{4}$ Numerical results for the throughput, which are not included here due to space limitation, show that the gain associated with the rate $R_{1}$ optimization, over Rayleigh channel, is small especially for values of $M \leq 9$.
}

[29, Eq. (12)]. Note that by setting $R_{1}=C_{\epsilon}^{M}$, the throughput $C_{\epsilon}^{\mathrm{IR}, \mathrm{M}}$ of HARQ-IR is maximized which is confirmed by numerical results that are not included in this paper due to space limitation. From Fig. 7, it can be seen that the HARQIR throughput is larger than the HARQ-CC throughput. For instance, for an SNR of $30 \mathrm{~dB}$ and $M=15$, the use of HARQIR allows achieving a throughput gain of $3.45 \mathrm{bps} / \mathrm{Hz}$. Besides, at high SNR, the throughput of HARQ-IR increases with $M$, while the throughput of HARQ-CC is almost the same for all values of $M$ at high SNR. Even though the throughput of HARQ-IR is larger than the throughput of HARQ-CC, the implementation on hardware of the HARQ-IR scheme requires a larger buffer size and involves decoding algorithms with a larger complexity [17].

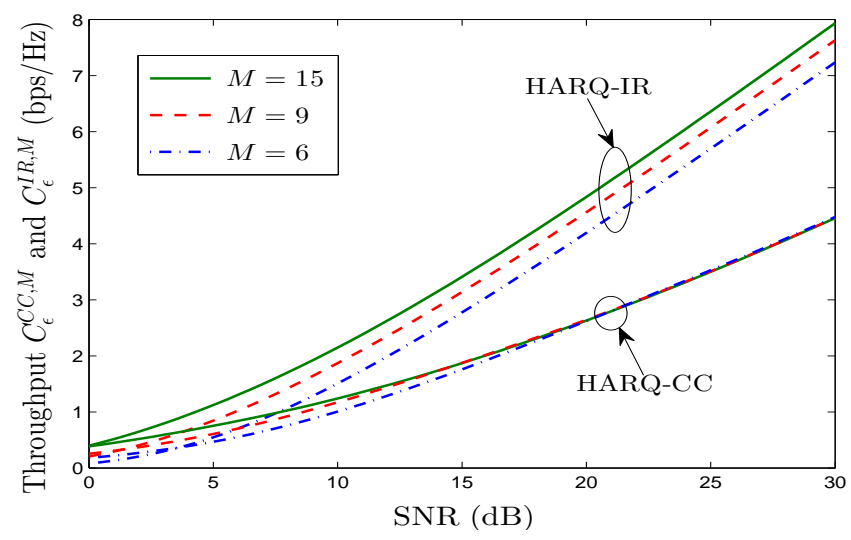

Fig. 7. Throughput of HARQ-CC and HARQ-IR over double Rayleigh channels for different values of $M$ and for $\epsilon=0.01$.

\section{B. Average Waiting Time and Sojourn Time}

In this section, the analytical expressions for the average waiting time $W$ and the sojourn time $T_{\text {soj }}$ provided in (32) and (36), respectively, are evaluated numerically and illustrated. These results have been obtained by setting the packet arrival rate $\lambda$ to 0.01 , while the frame duration is set to $T_{F}=0.1 \mathrm{~s}$.

Fig. 8 depicts the average waiting time $W$ versus the SNR for $M=6$ and 9 . We consider in this investigation two scenarios. In the first scenario, we set $R_{1}=\alpha^{*} C_{\epsilon}^{M}$, which is the rate that maximizes the throughput. For the second scenario, we set the rate $R_{1}=C_{\epsilon}^{M}(0 \mathrm{~dB})$, which is the value of $C_{\epsilon}^{M}$ evaluated at an SNR equal to $0 \mathrm{~dB}$. It can be observed from this figure that the average waiting time $W$ is larger for the first scenario compared to the second scenario. Actually, the rate for the first scenario is larger than the second one, which implies a larger average number of transmissions and consequently a larger waiting time $W$ for the first scenario.

Fig. 9 illustrates the sojourn time $T_{\text {soj }}$ versus the SNR for $M=10,15$, and 20 . The transmission rate $R_{1}$ per HARQ round is set to a constant value $R_{1}=1 \mathrm{bps}$. The choice of this value of $R_{1}$ implies that the same amount of data is being sent using HARQ-CC schemes with different values of $M$. Under this setting, the time required to successfully transmit a packet, $T_{\mathrm{soj}}$, decreases as the value of $M$ increases as shown in Fig. 9. In fact, for a constant value of $R_{1}$, as $M$ increases 


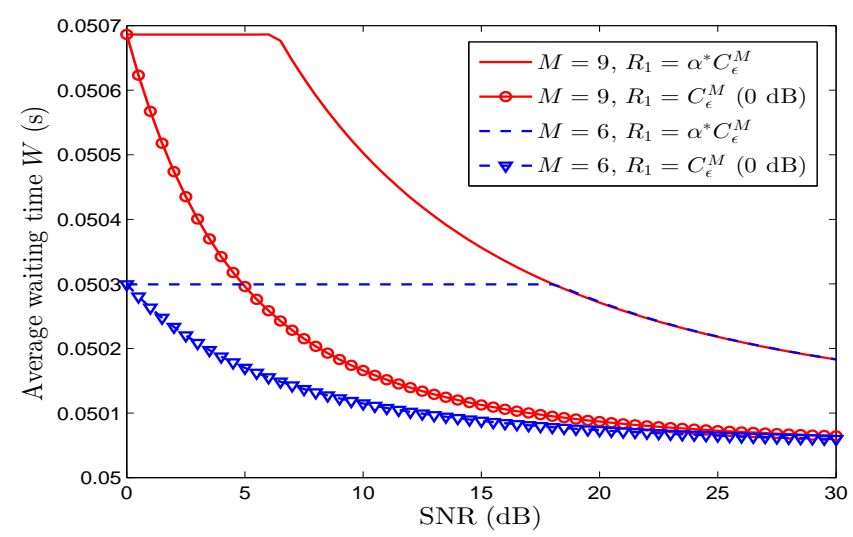

Fig. 8. Average waiting time $W$ of packets of HARQ-CC for the cases of $R_{1}=\alpha^{*} C_{\epsilon}^{M}$ and $R_{1}=C_{\epsilon}^{M}(0 \mathrm{~dB})$ rates per round.

the average number of transmissions to successfully deliver a packet $\mathbb{E}\left(T_{S}\right)$ decreases and consequently $T_{\text {soj }}$ drops.

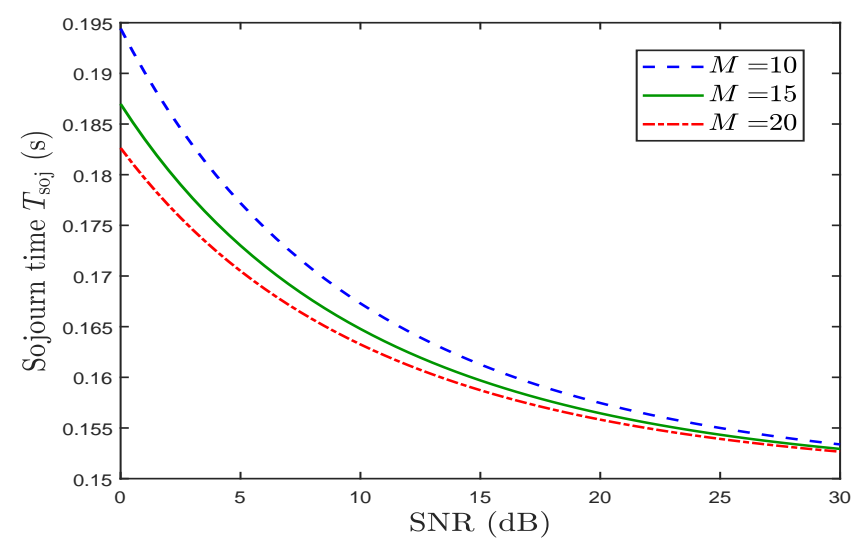

Fig. 9. Packet's sojourn time $T_{\text {soj }}$ in the network of queues of HARQ-CC for the cases of $R_{1}=\alpha^{*} C_{\epsilon}^{M}$ and $R_{1}=C_{\epsilon}^{M}(0 \mathrm{~dB})$ rates per round.

\section{Energy Efficiency and Average Consumed Power}

The energy efficiency $\eta_{E E}$ is illustrated as a function of the power $\mathcal{P}$ per HARQ round in Fig. 10 for different values of $M$. In our simulation, the rate $R_{1}$ per HARQ round has been set to $R_{1}=\alpha^{*} C_{\epsilon}^{M}$. It can be seen from Fig. 10 that the energy efficiency $\eta_{E E}$ decreases as the power $\mathcal{P}$ per HARQ round increases. As $M$ decreases, the energy efficiency $\eta_{E E}$ decays for low and medium values of $\mathcal{P}$, but for a value of $\mathcal{P}$ larger than $15 \mathrm{~dB}$ the energy efficiency $\eta_{E E}$ becomes the same for $M=5,6$ and 9. Using the expression of $\eta_{E E}$ in (44), we can see that $\eta_{E E}=\frac{C_{\epsilon}^{\mathbb{C C}, M}}{\mathcal{P}}$. Thus, when changing the value of $M$ only the throughput $C_{\epsilon}^{\mathrm{CC}, M}$ varies while the denominator term in $\eta_{E E}$ remains the same. The impact of $M$ on the throughput $C_{\epsilon}^{\mathrm{CC}, M}$ has been illustrated in Fig. 5. From Fig. 5, it can be seen that the throughput increases with $M$ for low and medium values of the SNR, while for high SNR values the throughput $C_{\epsilon}^{\mathrm{CC}, M}$ is the same for $M=5$, 6 , and 9. This fact explains the impact of $M$ on the energy efficiency curves in Fig. 10. Using (44), it can be concluded that the energy efficiency $\eta_{E E}$ tends to zero if the power $\mathcal{P}$

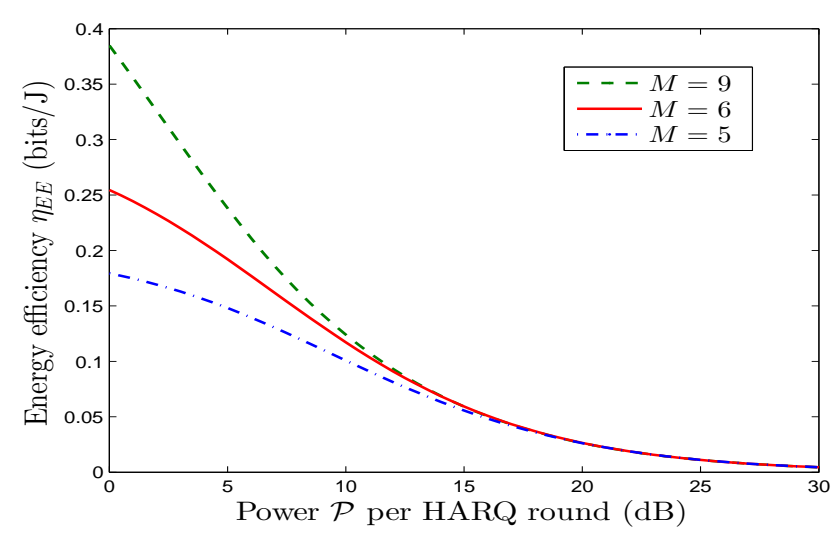

Fig. 10. Energy efficiency $\eta_{E E}$ of HARQ-CC versus the power $\mathcal{P}$ per HARQ round.

tends to infinity, as the throughput $C_{\epsilon}^{\mathrm{CC}, M}$ is upper bounded by the channel ergodic capacity.

To maximize the energy efficiency $\eta_{E E}$, we have to minimize the power $\mathcal{P}$, which results in a lower throughput $C_{\epsilon}^{\mathrm{CC}, M}$. Thus, there is a tradeoff between the throughput $C_{\epsilon}^{\mathrm{CC}, M}$ and the energy efficiency $\eta_{E E}$ as illustrated in Fig. 11. In this figure, we plot the energy efficiency $\eta_{E E}$ versus the throughput $C_{\epsilon}^{\mathrm{CC}, M}$ for different values of $M$. The tradeoff curves has

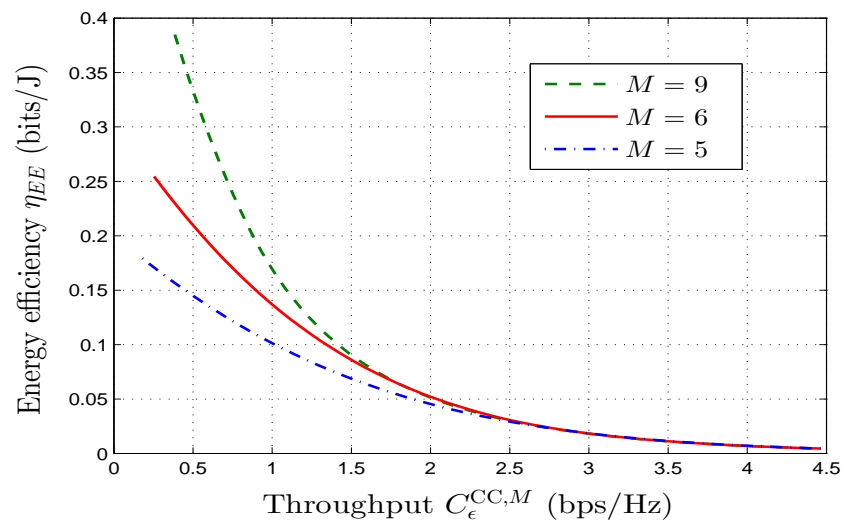

Fig. 11. Tradeoff between the energy efficiency $\eta_{E E}$ and the throughput $C_{\epsilon}^{\mathrm{CC}, M}$.

been obtained by setting the rate $R_{1}$ per HARQ round to $R_{1}=\alpha^{*} C_{\epsilon}^{M}$. From Fig. 11, it can be observed that if the target energy efficiency $\eta_{E E}$ increases, the achievable throughput $C_{\epsilon}^{\mathrm{CC}, M}$ decreases, for a fixed value of $M$. For instance, if the target $\eta_{E E}$ is increased from 0.1 bits $/ \mathrm{J}$ to $0.35 \mathrm{bits} / \mathrm{J}$, the achievable throughput $C_{\epsilon}^{\mathrm{CC}, M}$ decreases from $1.4 \mathrm{bps} / \mathrm{Hz}$ to $0.45 \mathrm{bps} / \mathrm{Hz}$ for $M=9$. Note that for a fixed target energy efficiency $\eta_{E E}$, the achievable throughput $C_{\epsilon}^{\mathrm{CC}, M}$ can be improved if $M$ is increased. For a fixed target throughput $C_{\epsilon}^{\mathrm{CC}, M}$, the energy efficiency $\eta_{E E}$ increases as $M$ increases. If the target throughput $C_{\epsilon}^{\mathrm{CC}, M}$ is larger than $2.5 \mathrm{bps} / \mathrm{Hz}$ then the variation of $M$ does not have any impact on the energy efficiency $\eta_{E E}$. 


\section{Impact of Imperfect Feedback}

In this section, we investigate the impact of imperfect feedback channels on the system performance by varying the feedback error probability $\epsilon_{f b}$. The analytical expressions for the average number of transmissions, the throughput, the average waiting time, and the sojourn time in the case of imperfect feedback channels presented in Sections V and VI are evaluated numerically and illustrated.

Fig. 12 presents the average number of transmissions $\mathbb{E}\left(T_{r}\right)$ of HARQ-CC with perfect and imperfect feedback channels. The maximum number of retransmissions $M$ has been set to 9 , whereas the transmission rate $R_{1}$ per HARQ round has been chosen as $R_{1}=\alpha^{*} C_{\epsilon}^{M}$. The feedback error probability $\epsilon_{f b}$ has been set to $0,0.3$, and 0.5. From Fig. 12, it can be seen that the average number of transmissions $\mathbb{E}\left(T_{r}\right)$ drops as the feedback error probability $\epsilon_{f b}$ increases, especially for low and medium values of the SNR. By examining the probability of

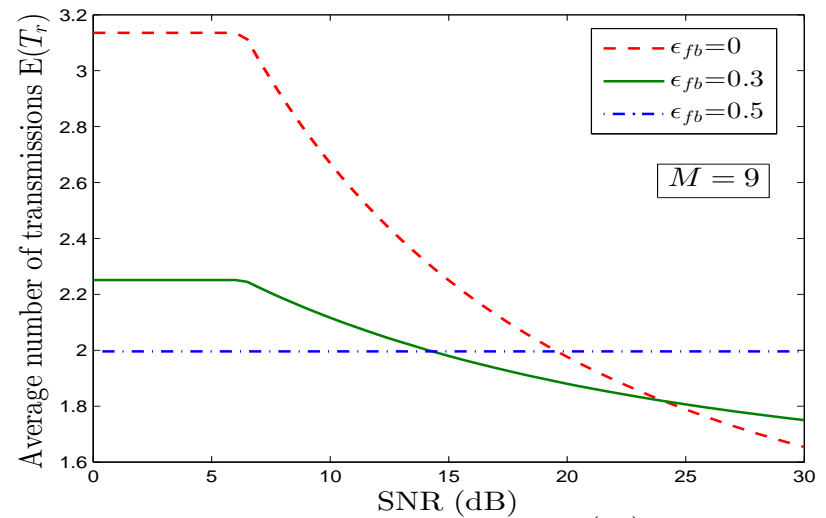

Fig. 12. The average number of transmissions $\mathbb{E}\left(T_{r}\right)$ with perfect and imperfect feedback channels.

packet loss $\xi_{M}$ [see (29)] for different values of $\epsilon_{f b}$, we notice that $\xi_{M}$ increases as $\epsilon_{f b}$ increases. For instance, for an SNR of $0 \mathrm{~dB}$, the packet loss probability $\xi_{M}$ is in the order of 0.01 , 0.43 , and 0.58 for $\epsilon_{f b}$ equal to $0,0.3$, and 0.5 , respectively. By analyzing the expression of $\xi_{M}$ [see (29)], it turns out that a high value (larger than 0.3 ) of the packet loss probability $\xi_{M}$ indicates that the transmitted packets are lost after the very first rounds (i.e., 1st, 2nd, 3rd, or 4th rounds mainly). This fact explains that the average number of transmissions $\mathbb{E}\left(T_{r}\right)$ drops as the feedback error probability $\epsilon_{f b}$ increases. As the SNR increases, $\mathbb{E}\left(T_{r}\right)$ decays for $\epsilon_{f b}$ equal to 0 and 0.3 . However, we notice that the average number of transmissions $\mathbb{E}\left(T_{r}\right)$ remains constant for all SNR values if the feedback error probability $\epsilon_{f b}$ equals 0.5 . At an SNR of $30 \mathrm{~dB}$, we observe in Fig. 12 that $\mathbb{E}\left(T_{r}\right)$ increases as $\epsilon_{f b}$ increases. The reason behind this are ACK $\rightarrow$ NACK errors.

In Fig. 13, we plot the throughput $C_{\epsilon}^{\mathrm{Imper}, \mathrm{M}}$ with perfect and imperfect feedback channels for different values of $\epsilon_{f b}$ and for $M=9$. The transmission rate $R_{1}$ per HARQ round has been set to $R_{1}=\alpha^{*} C_{\epsilon}^{M}$. From this figure, it can be observed that the throughput $C_{\epsilon}^{\text {Imper,M }}$ drops as the feedback error probability $\epsilon_{f b}$ increases. From the expression of the throughput $C_{\epsilon}^{\text {Imper,M }}$ in (30), it can be concluded that $C_{\epsilon}^{\text {Imper,M }}$ decreases if the packet loss probability $\xi_{M}$ increases or if $\mathbb{E}\left(T_{r}\right)$ increases. Our investigation has shown that $\xi_{M}$

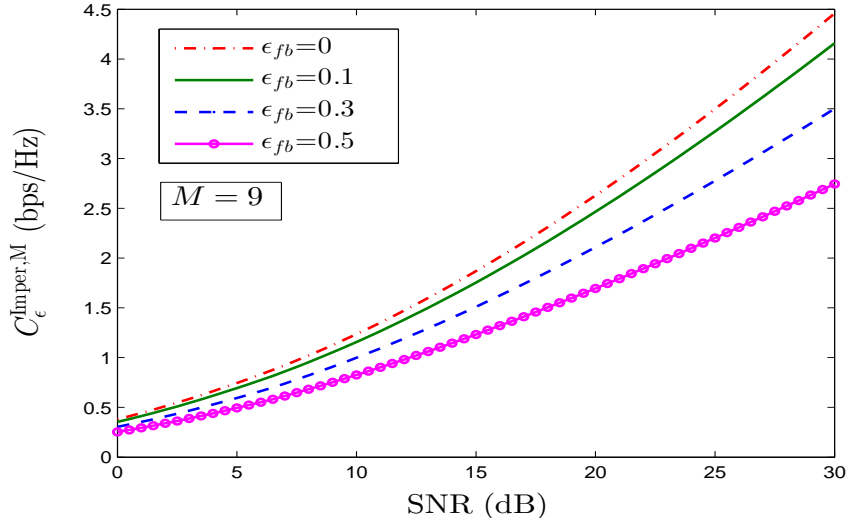

Fig. 13. Throughput $C_{\epsilon}^{\text {Imper,M }}$ with perfect and imperfect feedback channels.

increases as $\epsilon_{f b}$ increases, while $\mathbb{E}\left(T_{r}\right)$ drops for low and medium SNR values and increases at high SNR values as $\epsilon_{f b}$ increases. Globally, the trend of the throughput $C_{\epsilon}^{\text {Imper,M }}$ is governed by its numerator which drops as $\epsilon_{f b}$ increases, leading to the decay of $C_{\epsilon}^{\mathrm{Imper}, \mathrm{M}}$ with the increase of the feedback error probability $\epsilon_{f b}$. For example, for an SNR of $30 \mathrm{~dB}$, the throughput $C_{\epsilon}^{\mathrm{Imper}, \mathrm{M}}$ drops from $4.5 \mathrm{bps} / \mathrm{Hz}$ to $3.5 \mathrm{bps} / \mathrm{Hz}$ and $2.7 \mathrm{bps} / \mathrm{Hz}$, if the feedback error probability $\epsilon_{f b}$ increases from 0 to 0.3 and 0.5 , respectively.

Fig. 14 illustrates the average waiting time $W_{I}$ for different values of the feedback error probability $\epsilon_{f b}$. The value of $M$ has been set to 9 , the rate $R_{1}$ per HARQ round is equal to $R_{1}=\alpha^{*} C_{\epsilon}^{M}$. We can observe from Fig. 14 that the average waiting time $W_{I}$ drops as the feedback error probability $\epsilon_{f b}$ increases.

Fig. 15 depicts the sojourn time $T_{\mathrm{soj}, I}$ for different values of the feedback error probability $\epsilon_{f b}$. The value of $M$ has been set to 15 , the rate $R_{1}$ per HARQ round has a constant value with respect to the SNR. From Fig. 15, it can be observed that as the feedback error probability $\epsilon_{f b}$ increases the sojourn time $T_{\mathrm{soj}, I}$ increases. Note that the sojourn time represents the required time for successful packet delivery. As $\epsilon_{f b}$ increases $\mathbb{E}\left(T_{S, I}\right)$ increases and consequently $T_{\mathrm{soj}, I}$ increases.

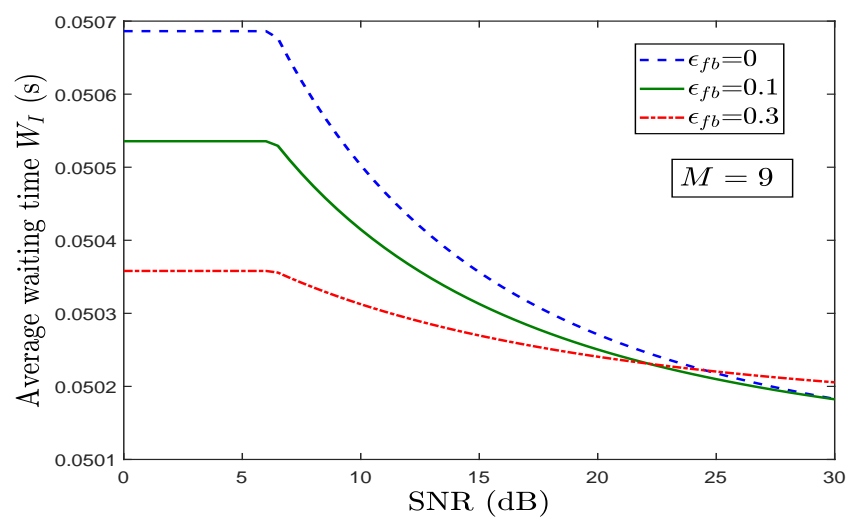

Fig. 14. Average waiting time $W_{I}$ of packets for different values of feedback error probabilities $\epsilon_{f b}$. 


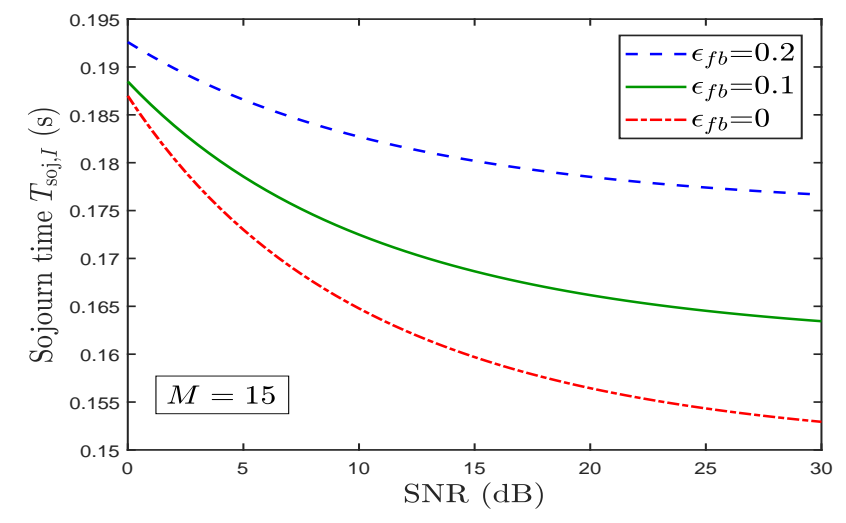

Fig. 15. Packet's sojourn time $T_{\mathrm{soj}, I}$ in the network of queues for different values of feedback error probabilities $\epsilon_{f b}$.

\section{CONCLUSION}

In this paper, we investigated the performance of HARQ-CC over double Rayleigh channels from an information theoretic perspective. In our analysis, the transmission rate is adjusted to the average SNR such that a fixed target outage probability is not exceeded which ensures link reliability. We provided analytical expressions characterizing the statistical properties of double Rayleigh channels, such as the PDF of the instantaneous SNR, the outage probability, and the ergodic capacity.

Generic expressions are derived for the $\epsilon$-outage capacity, the average number of transmissions, and the throughput of HARQ-CC, which are valid for any fading channel. Afterwards, analytical expressions of the throughput of HARQ-CC over Rayleigh and double Rayleigh channels are obtained. We formulated the throughput optimization problem and determined the optimal rate per round such that the throughput of HARQ-CC is maximized. Moreover, we investigated the delay experienced by Poisson arriving packets and derived analytical expressions for the average waiting time and the average sojourn time in the buffer when using HARQ-CC. We considered in our analysis perfect and imperfect feedback channels.

Our analysis has revealed that HARQ-CC allows improving the achievable communication rate compared to fixed time diversity schemes. To maximize the throughput of HARQ$\mathrm{CC}$, the rate per HARQ round should be less than the rate required to meet the outage constraint. Our comparison of the performance of HARQ-CC over Rayleigh and double Rayleigh channels shows that double Rayleigh channels have a higher severity of fading and result in a larger degradation of the throughput. Our analysis demonstrated that HARQ-IR achieves a larger throughput compared to HARQ-CC, while HARQ-CC is simpler to implement, has a lower decoding complexity, and requires less memory resources. In the case of imperfect feedback channels, the increase of the feedback error probability results in the degradation of the system throughput due to the increase of packet loss. Our analysis revealed that it is not possible to jointly maximize the energy and the throughput of HARQ-CC. Therefore, we have explored the tradeoff between the energy efficiency and the throughput of this scheme.

This work can serve as a framework for designing and optimizing communication systems with double Rayleigh fading. There are several communication scenarios for which the underlying fading follows a double Rayleigh distribution. These scenarios include urban vehicle-to-vehicle communication systems, amplify-and-forward relaying, as well as keyhole channels.

\section{APPENDIX A \\ ProOf OF (24)}

In this appendix, we derive an expression for the PDF and the CDF of a sum of squared double Rayleigh processes $\chi_{m}^{s}=\sum_{i=1}^{m} \chi_{i}$. It is well known [25] that the PDF of a sum of squared Rayleigh processes is given by the chi-square distribution. However, no closed-form solution exists for the sum of squared double Rayleigh processes. To cope with this problem, we use the Laguerre series [33] to approximate the PDF $p_{\chi_{m}^{s}}(x)$ of $\chi_{m}^{s}$ as

$$
p_{\chi_{m}^{s}}(x) \approx p_{0}^{\chi_{m}^{s}}(x)=\frac{x^{\alpha_{\chi_{m}^{s}}}}{\beta_{\chi_{m}^{s}}^{\left(\alpha_{\chi_{m}^{s}}+1\right)} \Gamma\left(\alpha_{\chi_{m}^{s}}+1\right)} \exp \left(-\frac{x}{\beta_{\chi_{m}^{s}}}\right)
$$

where $\alpha_{\chi_{m}^{s}}=\frac{\left[\mathbb{E}\left(\chi_{m}^{s}\right)\right]^{2}}{\operatorname{Var}\left(\chi_{m}^{s}\right)}-1$ and $\beta_{\chi_{m}^{s}}=\frac{\operatorname{Var}\left(\chi_{m}^{s}\right)}{\mathbb{E}\left(\chi_{m}^{s}\right)}$. A similar procedure was used in [34] to determine the PDF of the sum of double Rice processes. In order to evaluate the terms $\alpha_{\chi_{m}^{s}}$ and $\beta_{\chi_{m}^{s}}$, we need to determine the mean value and variance of the process $\chi_{m}^{s}$. For the mean value of $\chi_{m}^{s}$, the following equality holds $\mathbb{E}\left(\chi_{m}^{s}\right)=\sum_{i=1}^{m} \mathbb{E}\left(\chi_{i}\right)=m \mathbb{E}(\chi)$. The mean value of $\chi$ can be obtained as

$$
\mathbb{E}(\chi)=\int_{0}^{\infty} \chi p_{\chi}(\chi) d \chi=\int_{0}^{\infty} \frac{2 \chi}{\bar{\chi}} K_{0}\left(2 \sqrt{\frac{\chi}{\bar{\chi}}}\right) d \chi .
$$

Using the change of variable $u=\sqrt{\frac{\chi}{\bar{\chi}}}$ and [19, Eq. (6561.16)], the mean value of $\chi$ reads as

$$
\mathbb{E}(\chi)=\int_{0}^{\infty} 4 u^{3} \bar{\chi} K_{0}(2 u) d u=\bar{\chi} .
$$

As the processes $\chi_{i}$ are i.i.d., the variance of the process $\chi_{m}^{s}$ is expressed as

$$
\operatorname{Var}\left(\chi_{m}^{s}\right)=\sum_{i=1}^{m} \operatorname{Var}\left(\chi_{i}\right)=m\left\{\mathbb{E}\left(\chi^{2}\right)-\mathbb{E}(\chi)^{2}\right\}
$$

with

$$
\mathbb{E}\left(\chi^{2}\right)=\int_{0}^{\infty} \bar{\chi}^{2} 4 u^{5} K_{0}(2 u) d u=4 \bar{\chi}^{2} .
$$

Hence, we get $\operatorname{Var}\left(\chi_{m}^{s}\right)=3 m \bar{\chi}^{2}$. It follows that the parameters $\alpha_{\chi_{m}^{s}}$ and $\beta_{\chi_{m}^{s}}$ are given as $\alpha_{\chi_{m}^{s}}=\frac{\left[\mathbb{E}\left(\chi_{m}^{s}\right)\right]^{2}}{\operatorname{Var}\left(\chi_{m}^{s}\right)}-1=\frac{m}{3}-1$ and $\beta_{\chi_{m}^{s}}=\frac{\operatorname{Var}\left(\chi_{m}^{s}\right)}{\mathbb{E}\left(\chi_{m}^{s}\right)}=3 \bar{\chi}$. After determining the PDF $p_{\chi_{m}^{s}}(x)$ of $\chi_{m}^{s}$, the corresponding CDF $F_{\chi_{m}^{s}}(x)$ is deduced as

$$
F_{\chi_{m}^{s}}(x) \approx \int_{0}^{x} p_{0}^{\chi_{m}^{s}}(u) d u=\Gamma_{\alpha_{\chi_{m}^{s}}+1}\left(\frac{x}{\beta_{\chi_{m}^{s}}}\right)
$$

where $\Gamma_{a}(\cdot)$ is the incomplete gamma function [22, Eq. (6.5.1)]. 


\section{APPENDIX B}

PROOF OF (31)

In this appendix, we derive the expression of the average number of transmissions with imperfect feedback. We distinguish between two cases: (i) The transmission of a packet terminates after $m$ rounds with $1 \leq m \leq M-1$. (ii) The transmission terminates after $M$ rounds.

The transmission of a packet terminates after $m$ rounds $(1 \leq$ $m \leq M-1)$ if:

- There is a reception failure in the first $m$ rounds, a NACK $\rightarrow$ ACK error occurs in the $m$ th round, whereas the first $m-1$ NACKs are received correctly. This event happens with a probability equal to $P\left(F^{1}, \ldots, F^{m}\right)(1-$ $\left.\epsilon_{f b}\right)^{m-1} \epsilon_{f b}$.

- There is a successful reception of a packet in the $j$ th round $(1 \leq j \leq m)$, but an ACK is only received in the $m$ th round due to imperfect feedback. That is, a NACK is correctly received for rounds 1 to $j-1$, then an ACK $\rightarrow$ NACK error occurs for rounds $j, \ldots, m$, and finally an ACK is correctly received in round $m$ and the transmission of the packet terminates. These events occur with a probability equal to $\sum_{j=1}^{m} P\left(F^{1}, \ldots, F^{j-1}, S^{j}\right)\left(1-\epsilon_{f b}\right)^{j} \epsilon_{f b}^{m-j}$, where $P\left(F^{1}, \ldots, F^{j-1}, S^{j}\right)$ denotes the probability of a successful reception in round $j$. Note that this success probability is expressed as $P\left(F^{1}, \ldots, F^{j-1}, S^{j}\right)=$ $P\left(F^{1}, \ldots, F^{j-1}\right)-P\left(F^{1}, \ldots, F^{j}\right)$, while if $j=1$ the probability of a successful reception after the first round $P\left(S^{1}\right)=1-P\left(F^{1}\right)$. Using these identity, we can write $\sum_{j=1}^{m} P\left(F^{1}, \ldots, F^{j-1}, S^{j}\right)\left(1-\epsilon_{f b}\right)^{j} \epsilon_{f b}^{m-j}=$

$\left[1-P\left(F^{1}\right)\right]\left(1-\epsilon_{f b}\right) \epsilon_{f b}^{m-1}$

$+\sum_{j=2}^{m}\left[P\left(F^{1}, \ldots, F^{j-1}\right)-P\left(F^{1}, \ldots, F^{j}\right)\right](1$ $\left.\epsilon_{f b}\right)^{j} \epsilon_{f b}^{m-j}$.

Note that the above events are mutually exclusive. Thus, we can sum the probabilities of these events. The second possibility is that the transmission of a packet terminates after $M$ rounds which takes place if:

- There is a reception failure of a packet after $M-1$ rounds with $M-1$ correct NACKs, regardless of what happens in the $M$ th round. This event happens with a probability equal to $P\left(F^{1}, \ldots, F^{M-1}\right)\left(1-\epsilon_{f b}\right)^{M-1}$.

- There is a successful reception in the $j$ th round $(1 \leq j \leq M-1)$, but a NACK is received until round $M-1$ due to an imperfect feedback channel. In this case, we have $j-1$ reception failures with correct NACK, then a successful reception in round $j$, and an ACK $\rightarrow$ NACK error occurs for rounds $j, \ldots, M-1$. These events occur with a probability equal to $\sum_{j=1}^{M-1} P\left(F^{1}, \ldots, F^{j-1}, S^{j}\right)\left(1-\epsilon_{f b}\right)^{j-1} \epsilon_{f b}^{M-j}$, which is expressed in terms of reception failure probability as $\left[1-P\left(F^{1}\right)\right] \epsilon_{f b}^{M-1}+$ $\sum_{j=2}^{M-1}\left[P\left(F^{1}, \ldots, F^{j-1}\right)-P\left(F^{1}, \ldots, F^{j}\right)\right](1$ $\left.\epsilon_{f b}\right)^{j-1} \epsilon_{f b}^{M-j}$.

All the above events are mutually exclusive. Therefore, the average number of transmissions with imperfect feedback is obtained by summing up the probability of these events weighted by the number of transmissions associated with each event. Thus, we can express the average number of transmissions with imperfect feedback channel as

$$
\begin{aligned}
& \mathbb{E}\left(T_{r, I}\right)=\sum_{m=1}^{M-1} m\left(P\left(F^{1}, \ldots, F^{m}\right)\left(1-\epsilon_{f b}\right)^{m-1} \epsilon_{f b}\right. \\
& +\left[1-P\left(F^{1}\right)\right]\left(1-\epsilon_{f b}\right) \epsilon_{f b}^{m-1}+\sum_{j=2}^{m}\left(1-\epsilon_{f b}\right)^{j} \epsilon_{f b}^{m-j} \\
& \left.\cdot\left[P\left(F^{1}, \ldots, F^{j-1}\right)-P\left(F^{1}, \ldots, F^{j}\right)\right]\right)+M\left(\left(1-\epsilon_{f b}\right)^{M-1}\right. \\
& \cdot P\left(F^{1}, \ldots, F^{M-1}\right)+\left[1-P\left(F^{1}\right)\right] \epsilon_{f b}^{M-1}+\sum_{j=2}^{M-1} \epsilon_{f b}^{M-j} \\
& \left.\left.\cdot\left(1-\epsilon_{f b}\right)^{j-1}\left[P\left(F^{1}, \ldots, F^{j-1}\right)-P\left(F^{1}, \ldots, F^{j}\right)\right]\right), \text { (B.1 }\right)
\end{aligned}
$$

which is the desired final result.

\section{APPENDIX C \\ PROOF OF (37)}

In this appendix, we derive the expression of the average number of transmissions for successfully received packets $\mathbb{E}\left(T_{S}\right)$ in the case of perfect feedback channels. The random variable $T_{S}$ accounts for the number of transmissions to successfully receive a packet. The random variable $T_{S}$ is discrete with values in the set $\{1, \ldots, m, \ldots, M\}$ :

- $T_{S}=1$, if the transmission of the packet succeeds in the first round. We denote the probability of this event by $P\left(S^{1}\right)$. The probability of a failure after one HARQ round is referred to as $P\left(F^{1}\right)$. Note that $P\left(S^{1}\right)=1-$ $P\left(F^{1}\right)$.

- $T_{S}=m$, if the transmission of the packet succeeds in the $m$ th round. We denote the probability of this event by $P\left(F^{1}, \ldots, F^{m-1}, S^{m}\right)$. The probability of a failure after $m$ HARQ rounds is referred to as $P\left(F^{1}, \ldots, F^{m-1}, F^{m}\right)$. Note that $P\left(F^{1}, \ldots, F^{m-1}, S^{m}\right)=P\left(F^{1}, \ldots, F^{m-1}\right)-$ $P\left(F^{1}, \ldots, F^{m-1}, F^{m}\right)$.

- $T_{S}=M$, if the transmission of the packet succeeds in the $M$ th round. We denote the probability of this event by $P\left(F^{1}, \ldots, F^{m-1}, S^{M}\right)$. The probability of a failure after $M$ HARQ rounds is referred to as $P\left(F^{1}, \ldots, F^{m-1}, F^{M}\right)$. Note that $P\left(F^{1}, \ldots, F^{M-1}, S^{M}\right)=P\left(F^{1}, \ldots, F^{M-1}\right)-$ $P\left(F^{1}, \ldots, F^{M-1}, F^{M}\right)$.

Thus, the average number of successful transmissions can be obtained as

$$
\begin{aligned}
& \mathbb{E}\left(T_{S}\right)=1 P\left(S^{1}\right)+\sum_{m=2}^{M} m P\left(F^{1}, \ldots, F^{m-1}, S^{m}\right) \\
& =1 P\left(S^{1}\right)+\sum_{m=2}^{M} m P\left(F^{1}, \ldots, F^{m-1}, S^{m}\right) \\
& +M P\left(F^{1}, \ldots, F^{M-1}, F^{M}\right)-M P\left(F^{1}, \ldots, F^{M-1}, F^{M}\right)
\end{aligned}
$$




$$
\begin{aligned}
& =1+\sum_{m=1}^{M-1} P\left(F^{1}, \ldots, F^{m}\right)-M P\left(F^{1}, \ldots, F^{M-1}, F^{M}\right) \\
& =\mathbb{E}\left(T_{r}\right)-M P\left(F^{1}, \ldots, F^{M-1}, F^{M}\right) .
\end{aligned}
$$

\section{REFERENCES}

[1] A. G. Zajić, G. L. Stüber, and T. G. Pratt, "Statistical modelling and experimental verification for wideband MIMO mobile-to-mobile channels in urban environments," in Proc. 15th International Conference on Telecommunications 2008, St.Petersburg, Russia, Jun. 2008, pp. 1-6.

[2] M. Pätzold, B. Hogstad, and N. Youssef, "Modeling, analysis, and simulation of MIMO mobile-to-mobile fading channels," IEEE Transactions on Wireless Communications, vol. 7, no. 2, pp. 510-520, Feb. 2008.

[3] A. Chelli and M. Pätzold, "The impact of fixed and moving scatterers on the statistics of MIMO vehicle-to-vehicle channels," in Proc. VTC Spring 2009 - IEEE 69th Vehicular Technology Conference, Barcelona, Spain, Apr. 2009, pp. 1-6.

[4] H. Zhiyi, C. Wei, Z. Wei, M. Pätzold, and A. Chelli, "Modelling of MIMO vehicle-to-vehicle fading channels in T-junction scattering environments," in Proc. 3rd European Conference on Antennas and Propagation, 2009. EuCAP 2009., Berlin, Germany, Mar. 2009, pp. 652 656.

[5] F. Yilmaz and M. S. Alouini, "Product of the powers of generalized Nakagami-m variates and performance of cascaded fading channels," in IEEE Global Telecommunications Conference (GLOBECOM 2009), Nov 2009, pp. 1-8.

[6] D. Chizhik, G. J. Foschini, M. J. Gans, and R. A. Valenzuela, "Keyholes, correlations, and capacities of multielement transmit and receive antennas," IEEE Transactions on Wireless Communications, vol. 1, no. 2, pp. 361-368, Apr 2002.

[7] J. Salo, H. M. El-Sallabi, and P. Vainikainen, "Statistical analysis of the multiple scattering radio channel," IEEE Transactions on Antennas and Propagation, vol. 54, no. 11, pp. 3114-3124, Nov 2006.

[8] J. Salo, H. El-Sallabi, and P. Vainikainen, "Impact of double-Rayleigh fading on system performance," in Proc. 1st International Symposium on Wireless Pervasive Computing, 2006, Jan. 2006, pp. 1-5.

[9] D. Costello, J. Hagenauer, H. Imai, and S. Wicker, "Applications of error-control coding," IEEE Transactions on Information Theory, vol. 44, no. 6, pp. 2531-2560, Oct. 1998.

[10] E. Dahlman, S. Parkvall, J. Skold, and P. Beming, $3 G$ Evolution: HSPA and LTE for Mobile Broadband. Academic Press, 2007.

[11] D. Chase, "Code combining-A maximum-likelihood decoding approach for combining an arbitrary number of noisy packets," IEEE Transactions on Communications, vol. 33, no. 5, pp. 385-393, May 1985.

[12] G. Caire and D. Tuninetti, "The throughput of hybrid-ARQ protocols for the Gaussian collision channel," IEEE Transactions on Information Theory, vol. 47, no. 5, pp. 1971-1988, Jul. 2001.

[13] H. E. Gamal, G. Caire, and M. O. Damen, "The MIMO ARQ channel: Diversity-multiplexing-delay tradeoff," IEEE Transactions on Information Theory, vol. 52, no. 8, pp. 3601-3621, Aug. 2006.

[14] Z. Shi, H. Ding, S. Ma, and K. W. Tam, "Analysis of HARQ-IR over time-correlated Rayleigh fading channels," IEEE Transactions on Wireless Communications, vol. 14, no. 12, pp. 7096-7109, Dec. 2015.

[15] P. Larsson, L. K. Rasmussen, and M. Skoglund, "Throughput analysis of ARQ schemes in Gaussian block fading channels," IEEE Transactions on Communications, vol. 62, no. 7, pp. 2569-2588, Jul. 2014.

[16] D. To, H. X. Nguyen, Q. T. Vien, and L. K. Huang, "Power allocation for HARQ-IR systems under QoS constraints and limited feedback," IEEE Transactions on Wireless Communications, vol. 14, no. 3, pp. 15811594, Mar. 2015.

[17] A. Chelli and M. S. Alouini, "On the performance of hybrid-ARQ with incremental redundancy and with code combining over relay channels," IEEE Transactions on Wireless Communications, vol. 12, no. 8, pp. 3860-3871, Aug. 2013.

[18] P. Wu and N. Jindal, "Performance of hybrid-ARQ in block-fading channels: A fixed outage probability analysis," IEEE Transactions on Communications, vol. 58, no. 4, pp. 1129-1141, Apr. 2010.

[19] I. S. Gradshteyn and I. M. Ryzhik, Table of Integrals, Series, and Products, Fifth Edition. Academic Press, 1994.

[20] W. Wongtrairat and P. Supnithi, "Performance of digital modulation in double Nakagami-m fading channels with MRC diversity," IEICE Transactions on Communications, vol. E92-B, no. 2, pp. 559-566, Feb. 2009.
[21] Y. L. Luke, The Special Functions and Their Approximations. Academic Press, 1969.

[22] M. Abramowitz and I. A. Stegun, Handbook of Mathematical Functions: with Formulas, Graphs, and Mathematical Tables. Courier Dover Publications, 1964.

[23] W. Rui and V. Lau, "Combined cross-layer design and HARQ for multiuser systems with outdated channel state information at transmitter (CSIT) in slow fading channels," IEEE Transactions on Wireless Communications, vol. 7, no. 7, pp. 2771-2777, Jul. 2008.

[24] S. Verdú and T. S. Han, "A general formula for channel capacity," IEEE Transactions on Information Theory, vol. 40, no. 4, pp. 1147-1157, Jul. 1994.

[25] M. K. Simon, Probability Distributions Involving Gaussian Random Variables: A Handbook for Engineers and Scientists. New York, USA: Springer, 2006.

[26] Wolfram Research, Mathematica Edition: Version 8.0. Champaign Illinois: Wolfram Research, Inc., 2010.

[27] A. Chelli and M. Pätzold, "On the performance of hybrid-ARQ with code combining over double Rayleigh fading channels," in Proc. IEEE 22nd Symposium on Personal, Indoor and Mobile Radio Communications (PIMRC 2011), Toronto, Canada, Sep. 2011, pp. 2014-2019.

[28] W. Chan, T.-C. Lu, and R.-J. Chen, "Pollaczek-Khinchin formula for the M/G/1 queue in discrete time with vacations," IEE Proceedings Computers and Digital Techniques, vol. 144, no. 4, pp. 222-226, Jul. 1997.

[29] A. Chelli, E. Zedini, M.-S. Alouini, J. R. Barry, and M. Pätzold, "Performance and delay analysis of hybrid ARQ with incremental redundancy over double Rayleigh fading channels," IEEE Transactions on Wireless Communications, vol. 13, no. 11, pp. 6245-6258, Nov. 2014

[30] H. Boujemâa, "Delay analysis of cooperative truncated HARQ with opportunistic relaying," IEEE Transactions on Vehicular Technology, vol. 58, no. 9, pp. 4795-4804, Nov 2009.

[31] C. Li, S. H. Song, J. Zhang, and K. B. Letaief, "Maximizing energy efficiency in wireless networks with a minimum average throughput requirement," in Proc. IEEE 2012 Wireless Communications and Networking Conference (WCNC 2012), Paris, France, Apr. 2012, pp. 11301134.

[32] G. Y. Li, Z. Xu, C. Xiong, C. Yang, S. Zhang, Y. Chen, and S. Xu, "Energy-efficient wireless communications: tutorial, survey, and open issues," IEEE Transactions on Wireless Communications, vol. 18, no. 6, pp. 28-35, Dec. 2011.

[33] S. Primak, V. Kontorovitch, and V. Lyandres, Stochastic Methods and their Applications to Communications: Stochastic Differential Equations Approach. Wiley, 2004.

[34] B. Talha and M. Pätzold, "On the statistical analysis of equal gain combining over multiple double Rice fading channels in cooperative networks," in 2010 IEEE 72nd Vehicular Technology Conference - Fall, Ottawa, Canada, Sep. 2010, pp. 1-5.

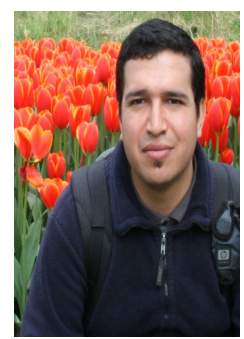

Ali Chelli (S'08, M'12) was born in Sfax, Tunisia. $\mathrm{He}$ received the B.Sc degree in communications from Ecole Superieure des Communications de Tunis (SUP'COM), in 2005. He received the M.Sc. degree in information and communication technology from University of Agder, Norway, in 2007. He received his $\mathrm{Ph} . \mathrm{D}$ degree in information and communication technology from University of Agder, Norway, in 2013. He was serving as a postdoctoral fellow in King Abdullah University of Science and Technology (KAUST). Afterwards, he joined the Department of Electronics and Telecommunications, Norwegian University of Science and Technology (NTNU), Trondheim as a researcher. Currently, he is a research scientist at the University of Agder, Norway. His research interests include machine learning for human activity detection and wireless communication theory with focus on performance analysis of cooperative relaying, vehicle-to-vehicle communications, game theory, and channel modelling. 


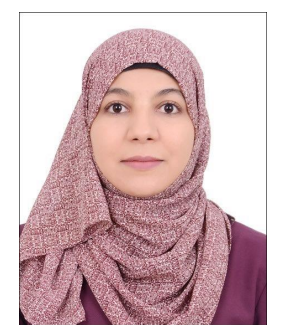

Emna Zedini (S'14, M'17) was born in Beja, Tunisia. She received the Diplome d'Ingenieur degree in telecommunication engineering and the M.Sc. degree from the Ecole Superieure des Communications de Tunis (SUP'COM), Tunis, Tunisia, in 2010 and 2011, respectively. She received the $\mathrm{Ph} . \mathrm{D}$. degree in Electrical Engineering from King Abdullah University of Science and Technology (KAUST), Saudi Arabia, in 2016. She joined recently Hamad Bin Khalifa University (HBKU), Doha, Qatar as a postdoctoral researcher. Her research interests include channel modeling and performance analysis of optical wireless communication systems, with current research focusing on underwater optical wireless communication.

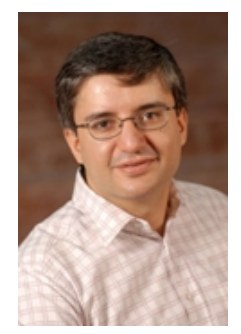

Mohamed-Slim Alouini (S'94, M'98, SM'03, F'09) was born in Tunis, Tunisia. He received the Ph.D. degree in Electrical Engineering from the California Institute of Technology (Caltech), Pasadena, CA, USA, in 1998. He served as a faculty member in the University of Minnesota, Minneapolis, MN USA, then in the Texas A\&M University at Qatar, Education City, Doha, Qatar before joining King Abdullah University of Science and Technology (KAUST), Thuwal, Makkah Province, Saudi Arabia as a Professor of Electrical Engineering in 2009. His current research interests include the modeling, design, and performance analysis of wireless communication systems.

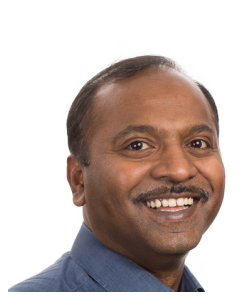

Ilangko Balasingham received the M.Sc. and Ph.D. degrees from the Department of Electronics and Telecommunications, Norwegian University of Science and Technology (NTNU), Trondheim, Norway in 1993 and 1998, respectively, both in signal processing. He performed his Master's degree thesis at the Department of Electrical and Computer Engineering, University of California Santa Barbara, USA. From 1998 to 2002, he worked as a Research Engineer developing image and video streaming solutions for mobile handheld devices at Fast Search \& Transfer ASA, Oslo, Norway, which is now part of Microsoft Inc. Since 2002 he has been with the Intervention Center, Oslo University Hospital, Oslo, Norway as a Senior Research Scientist, where he heads the Wireless Sensor Network Research Group. He was appointed as a Professor in Signal Processing in Medical Applications at NTNU in 2006. For the academic year 2016/2017 he was Professor by courtesy at the Frontier Institute, Nagoya Institute of Technology in Japan. His research interests include super robust short range communications for both in-body and on-body sensors, body area sensor network, microwave short range sensing of vital signs, short range localization and tracking mobile sensors, and nanoscale communication networks. He has authored or co-authored over 200 journal and conference papers, 7 book chapters, 42 abstracts, 5 patents, and 16 articles in popular press. Ilangko has given 16 invited/ keynotes at the international conferences. In addition he is active in organizing conferences (Steering Committee Member of ACM NANOCOM 2018-2021; General Chair: the 2019 IEEE Int. Symposium of Medical ICT, the 2012 Body Area Networks (BODYNETS) conference; TPC Chair of the 2015 ACM NANOCOM) and editorial board (Area Editor of Elsevier Nano Communication Networks 2013-unti now). $\mathrm{He}$ is a Senior IEEE member.

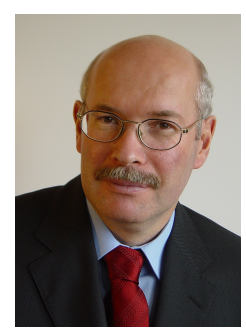

Matthias Pätzold was born in Engelsbach, Germany, in 1958. He received the Dipl.-Ing. and Dr.-Ing. degrees from Ruhr-University Bochum, Bochum, Germany, in 1985 and 1989, respectively, all in Electrical Engineering. In 1998, he received the habil. degree in Communications Engineering from the Technical University of Hamburg-Harburg, Hamburg, Germany. From 1990 to 1992, he was with ANT Nachrichtentechnik GmbH, Backnang, Germany, where he was engaged in digital satellite communications. From 1992 to 2001, he was with the Department of Digital Networks at the Technical University HamburgHarburg. Since 2001, he has been a full professor of mobile communications and the Head of the Mobile Communications Group at the University of Agder, Grimstad, Norway. He is the author of several books and more than 300 technical papers. His publications received 14 best paper awards. He has actively participated in numerous conferences, serving as a chair and a member of technical program committees. He served as an associate editor of IEEE Vehicular Technology Magazine from 2010 to 2017 and was a guest editor for several special issues in the field of mobile radio channels. Since 2017, he serves as a senior editor of the IEEE Vehicular Technology Magazine. $\mathrm{He}$ is a Senior Member of the IEEE. His current research interests include mobile radio communications, especially propagation and channel modelling, multiple-input multiple-output (MIMO) systems, cooperative communication systems, vehicular-to-vehicular communications, mobile-to-mobile communications, and coded-modulation techniques for fading channels. 